\title{
How to Avoid a Multirotor Flight Crash under Complete Propeller Motor Failures Based on State Variable Approach
}

\author{
Kaito Isogai, ${ }^{1 *}$ Hideo Nakano, ${ }^{2}$ and Hideaki Okazaki ${ }^{1}$ \\ ${ }^{1}$ Graduate School of Electrical and Information Engineering, Shonan Institute of Technology, \\ 1-1-25 Tsujido Nishikaigan, Fujisawa 251-8511, Japan \\ ${ }^{2}$ Department of Applied Computer Sciences, Shonan Institute of Technology, \\ 1-1-25 Tsujido Nishikaigan, Fujisawa 251-8511, Japan
}

(Received April 30, 2019; accepted August 19, 2019)

Keywords: multirotor, flight states to avoid a crash, motor failure problem, Euler angle state variable approach, flight operating points

In this paper, how to obtain motor speed signals for multirotor flight states to avoid a crash in the case of complete motor failures based on the Euler angle state variable approach is explained. First, the mathematical foundation for a multirotor as a rigid body is introduced. Secondly, state equations, maneuvers, and flight states (or flight operating points) of multirotors are summarized. Thirdly, in the case of complete motor failures, definitions of the motor speed control signal vector of the remaining motors and a method of directly providing motor speed control signals to achieve flight states to avoid a crash are provided. Finally, the method is applied to the problem of a multirotor vehicle experiencing motor failures. Numerical simulation results are also illustrated. In addition, the mathematical backgrounds of the Euler angle state equations of the multirotor and the proposed method are precisely explained in the appendices.

\section{Introduction}

Multirotors are aerial robotic vehicles that are increasing in popularity. ${ }^{(1-11)}$ These vehicles are currently being used in many applications such as surveillance and search and rescue missions. However, there is a potential risk to civil safety if a multirotor crashes, especially in an urban area. Therefore, it is important to consider multirotor flight control states in the case of complete motor failures. Representative related works are briefly discussed as follows. Mueller and D'Andrea presented a method based on Ref. 8 that proposed a method of allowing a quadrocopter to maintain its position in space after losing one, two (opposing), or three propellers by using the strategy of having the vehicle rotate freely about a fixed axis with respect to the body. ${ }^{(9)}$ Dongjie et al. proposed a method of analyzing the multicopter reliability related to an optimal design including i) a reliability analysis for multicopters with different numbers of rotors and ii) a reliability analysis for hexacopters with different rotor

*Corresponding author: e-mail: kaito.isogai.3@gmail.com

https://doi.org/10.18494/SAM.2019.2418 
configurations. ${ }^{(10)}$ Saied et al. presented a fault-tolerant control architecture including the use of a support vector machine (SVM) classification algorithm for the fault diagnosis of an octorotor after simultaneous or successive motor failures. ${ }^{(1)}$ However, how to obtain such motor control signals has not been fully established.

Hence, to clarify how to obtain such motor control signals, before considering the multirotor flight states to avoid a crash in the case of complete motor failures, on the basis of Refs. 12 and 13, we focus on reliable multirotor flight simulations and maneuverable flight controls by using an approach based on Euler angle rotational and translational state variables, which includes an analysis of the operating points. The main purpose of this study is to directly provide the motor speed signals for the multirotor flight states to avoid a crash in the case of complete motor failures based on the state variable approach.

In Sect. 2, we introduce the mathematical foundation for a multirotor as a rigid body, the multirotor body frame configurations, and the state equations, maneuvers, and flight states (or flight operating points) of the multirotor. In Sect. 3, in the case of complete motor failures, we provide definitions of the motor speed control signal vector of the remaining motors and a method of directly providing motor speed control signals to achieve flight states to avoid a crash. In Sect. 4, we apply the method to the problem of a multirotor vehicle experiencing motor failures and illustrate the simulation results. In Sect. 5, we summarize the principal results and describe future research.

In addition, we precisely explain the mathematical backgrounds of the preceding sections in the appendices. In Appendix 1, we describe dynamic system state equations for a multirotor as both a theorem of dynamic Euler angle state equations of rotations for a multirotor and a theorem of dynamic state equations of translations for the multirotor. In Appendix 2, we provide a theorem to achieve the multirotor maneuvers and flight states. In Appendix 3, in the case of complete motor failures, we provide two theorems to achieve the two types of multirotor flight states in Table 3 to avoid a crash.

In the following, $\mathbb{N}$ is the set of natural numbers, $\mathbb{R}$ is the set of real numbers, and $\mathbb{R}^{n}$ is the set of real number vectors. Matrices and vectors are indicated in bold. $\langle\cdot, \cdot\rangle$ denotes the scalar product, $[\cdot, \cdot]$ the vector product, $(\cdot)^{-1}$ the inverse matrix of $(\cdot)$, and $(\cdot)^{\mathrm{T}}$ the transposition of $(\cdot)$.

\section{Mathematical Description of a Multirotor}

In this section, we summarize the mathematical foundation for describing the motion of a multirotor as a rigid body that is based on Ref. 14. We also describe the multirotor body frame configurations. In addition, we provide dynamical Euler angle state equations of rotations for the multirotor and dynamical system state equations of translations for the multirotor, and we define the multirotor flight states and flight operating points.

\subsection{Rigid-body dynamics}

Table 1 shows the symbols for the motion of a multirotor as a rigid body based on Ref. 14. In addition, linear operator $\boldsymbol{B}$ is also described by a matrix form: 
Table 1

Mathematical description for the motion of a multirotor as a rigid body.

\begin{tabular}{|c|c|}
\hline Symbol & Description \\
\hline $\mathbb{R}^{3}$ & Three-dimensional real vector space \\
\hline$t \in \mathbb{R}$ & Time \\
\hline $\boldsymbol{w}: \boldsymbol{O}+\operatorname{span}\left\{\boldsymbol{e}_{1}, \boldsymbol{e}_{2}, \boldsymbol{e}_{3}\right\}^{(15)}$ & $\begin{array}{l}\text { Basis vectors of a right-handed Cartesian stationary coordinate system at the } \\
\text { origin } \boldsymbol{O} \text { (Fig. 1) }\end{array}$ \\
\hline $\begin{array}{l}\boldsymbol{W}: \boldsymbol{O} c+\operatorname{span}\left\{\boldsymbol{E}_{1}, \boldsymbol{E}_{2}, \boldsymbol{E}_{3}\right\} \\
\text { or } \boldsymbol{O}_{c}+\operatorname{span}\left\{\boldsymbol{e}_{1}, \boldsymbol{e}_{2}, \boldsymbol{e}_{3}\right\}^{(15)}\end{array}$ & $\begin{array}{l}\text { Basis vectors of a right-handed moving (or local) coordinate system connected to } \\
\text { the body at the center of mass } \boldsymbol{O}_{c}\end{array}$ \\
\hline B & Linear operator, $\boldsymbol{B}: W \rightarrow w$ \\
\hline $\boldsymbol{q} \in w$ & Radius vector of a point moving relative to the stationary system \\
\hline$Q(t)$ & Radius vector of the point relative to the moving system such that $\boldsymbol{q}=\boldsymbol{r}+\boldsymbol{B} \boldsymbol{Q}$ \\
\hline$\dot{q}$ & $\begin{aligned} \text { Absolute velocity such that } \dot{\boldsymbol{q}}= & \dot{\boldsymbol{r}}+\dot{\boldsymbol{B}} \boldsymbol{Q}+\boldsymbol{B} \dot{\boldsymbol{Q}}, \text { where an overdot represents time } \\
& \text { differentiation }\end{aligned}$ \\
\hline$r$ & $\begin{array}{l}\text { Radius vector of the moving coordinate system relative to the stationary } \\
\text { coordinate system }\end{array}$ \\
\hline$\dot{r}$ & Velocity of motion of the moving coordinate system \\
\hline$\Omega \in W$ & Vector of angular velocity in multirotors such that $\boldsymbol{\Omega}=\boldsymbol{B}^{\mathrm{T}} \boldsymbol{\omega}$ \\
\hline $\boldsymbol{\omega} \in w$ & Instantaneous angular velocity \\
\hline $\begin{array}{l}\hat{I}\left(I_{11} \neq 0, I_{22} \neq 0, I_{33} \neq 0, I_{i j}=0\right. \\
\quad \text { for } i \neq j)\end{array}$ & Moment of inertia for multirotors \\
\hline
\end{tabular}

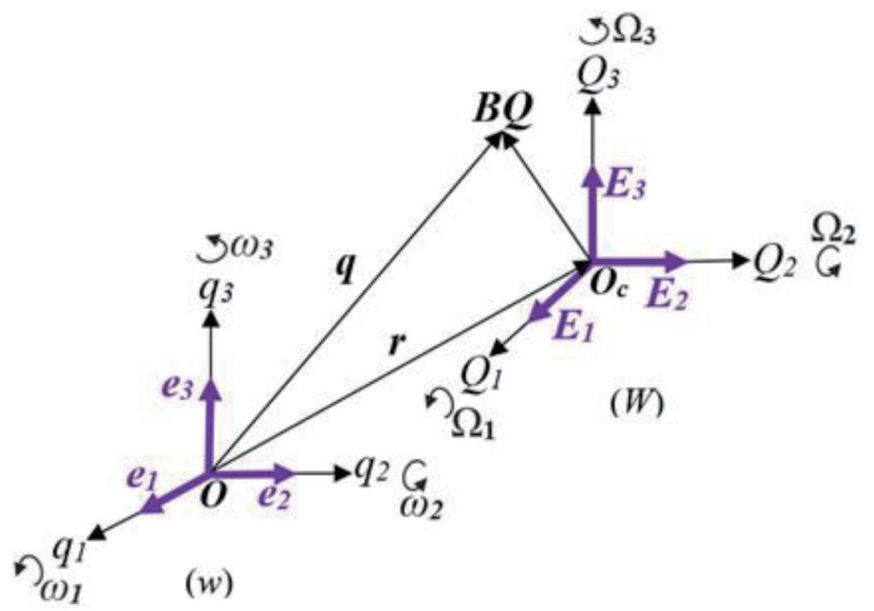

Fig. 1. (Color online) Radius vector of a point with respect to stationary $(w)$ and moving $(W)$ coordinate systems.

$$
\boldsymbol{B}=\left(\begin{array}{ccc}
\cos \psi \cos \theta & \cos \psi \sin \theta \sin \phi-\sin \psi \cos \phi & \cos \psi \sin \theta \cos \phi+\sin \psi \sin \phi \\
\sin \psi \cos \theta & \sin \psi \sin \theta \sin \phi+\cos \psi \cos \phi & \sin \psi \sin \theta \cos \phi-\cos \psi \sin \phi \\
-\sin \theta & \cos \theta \sin \phi & \cos \theta \cos \phi
\end{array}\right)
$$

The angles $\psi, \theta$, and $\phi$ are Tait-Bryan angles, which are examples of Euler angles. ${ }^{(12,13)}$ 


\subsection{Description of multirotor body frame configuration}

We assume that all rotors are the same, distributed evenly, and coplanar, and the distance from each rotor to the geometric center of the multirotor $\ell$ is equal. ${ }^{(10)}$ Each multirotor has a standard symmetrical configuration with a clockwise-rotating rotor adjacent to a counterclockwise-rotating rotor as shown in Figs. $2-4 . F_{i}(i=1,2, \ldots, 2 p, p=2,3,4)$ and $M_{i}$ $(i=1,2, \ldots, 2 p, p=2,3,4)$ in Figs. $2-4$ represent vertical forces and moments, respectively. Each motor of a multirotor has an angular speed $\omega_{M i}$ and produces a vertical force $F_{i}$ satisfying

$$
F_{i}=k_{F i} \omega_{M i}^{2}, \quad i=1,2, \ldots, 2 p, p=2,3,4
$$

Each motor also produces the moment

$$
M_{i}=k_{M i} \omega_{M i}^{2}, \quad i=1,2, \ldots, 2 p, p=2,3,4
$$

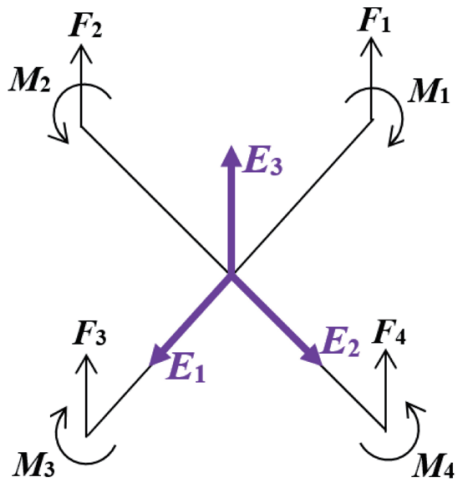

Fig. 2. (Color online) Quadrotor with standard configuration.

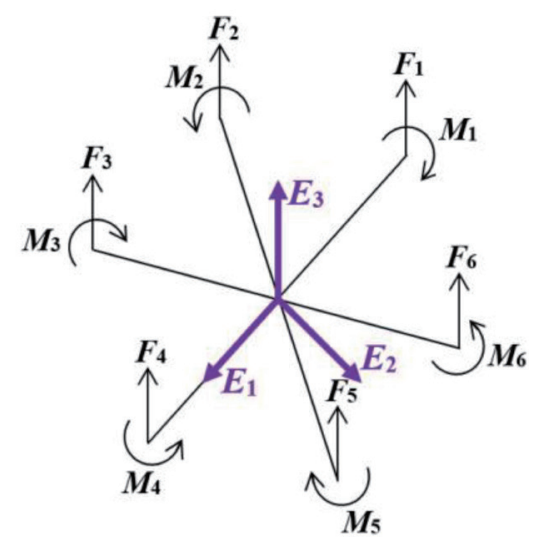

Fig. 3. (Color online) Hexarotor with standard configuration.

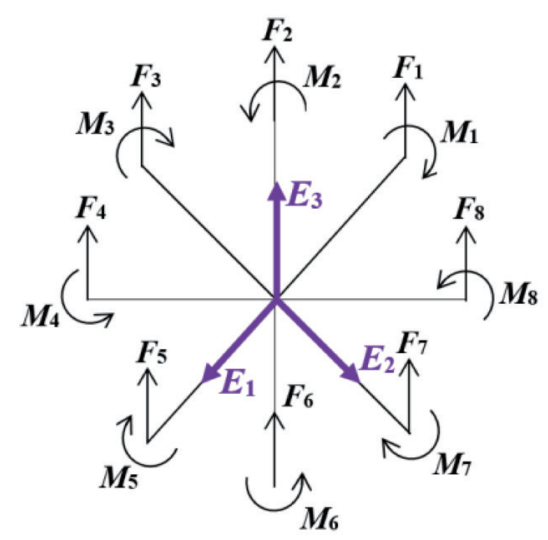

Fig. 4. (Color online) Octorotor with standard configuration. 
In practice, simple lumped parameter models are applied such that $k_{F}>0$ and $k_{M}>0$ are constants that can be easily determined from static thrust tests. For quadrotors $(p=2)$, hexarotors $(p=3)$, and octorotors $(p=4)$ that have standard symmetrical configurations, we define the moments of $\boldsymbol{S}_{\text {rot2p }} \boldsymbol{u}_{2 p}$ and the translational forces of $\boldsymbol{S}_{\text {tra2p }} \boldsymbol{u}_{2 p}, p=2,3,4$ as follows. For quadrotors $(p=2)$,

$$
\begin{gathered}
\boldsymbol{S}_{\mathrm{rot} 4}=\left(\begin{array}{cccc}
0 & -\ell \cdot k_{F 2} & 0 & \ell \cdot k_{F 4} \\
\ell \cdot k_{F 1} & 0 & -\ell \cdot k_{F 3} & 0 \\
-k_{M 1} & k_{M 2} & -k_{M 3} & k_{M 4}
\end{array}\right) \\
\boldsymbol{u}_{4}=\sum_{i=1}^{4} \omega_{M i}^{2} \boldsymbol{\varepsilon}_{M i} .
\end{gathered}
$$

For hexarotors $(p=3)$,

$$
\begin{gathered}
S_{\text {rot6 }}=\left(\begin{array}{cccccc}
0 & -\frac{\sqrt{3}}{2} \ell \cdot k_{F 2} & -\frac{\sqrt{3}}{2} \ell \cdot k_{F 3} & 0 & \frac{\sqrt{3}}{2} \ell \cdot k_{F 5} & \frac{\sqrt{3}}{2} \ell \cdot k_{F 6} \\
\ell \cdot k_{F 1} & 0.5 \ell \cdot k_{F 2} & -0.5 \ell \cdot k_{F 3} & -\ell \cdot k_{F 4} & -0.5 \ell \cdot k_{F 5} & 0.5 \ell \cdot k_{F 6} \\
-k_{M 1} & k_{M 2} & -k_{M 3} & k_{M 4} & -k_{M 5} & k_{M 6}
\end{array}\right), \\
\boldsymbol{u}_{6}=\sum_{i=1}^{6} \omega_{M i}^{2} \boldsymbol{\varepsilon}_{M i} .
\end{gathered}
$$

For octorotors $(p=4)$,

$$
\begin{gathered}
\boldsymbol{S}_{\mathrm{rot} 8}=\left(\begin{array}{cccccccc}
0 & -\frac{\sqrt{2}}{2} \ell \cdot k_{F 2} & -\ell \cdot k_{F 3} & -\frac{\sqrt{2}}{2} \ell \cdot k_{F 4} & 0 & \frac{\sqrt{2}}{2} \ell \cdot k_{F 6} & \ell \cdot k_{F 7} & \frac{\sqrt{2}}{2} \ell \cdot k_{F 8} \\
\ell \cdot k_{F 1} & \frac{\sqrt{2}}{2} \ell \cdot k_{F 2} & 0 & -\frac{\sqrt{2}}{2} \ell \cdot k_{F 4} & -\ell \cdot k_{F 5} & -\frac{\sqrt{2}}{2} \ell \cdot k_{F 6} & 0 & \frac{\sqrt{2}}{2} \ell \cdot k_{F 8} \\
-k_{M 1} & k_{M 2} & -k_{M 3} & k_{M 4} & -k_{M 5} & k_{M 6} & -k_{M 7} & k_{M 8}
\end{array}\right), \\
\boldsymbol{u}_{8}=\sum_{i=1}^{8} \omega_{M i}^{2} \boldsymbol{\varepsilon}_{M i} .
\end{gathered}
$$

For multirotors $(p=2,3,4)$,

$$
\begin{gathered}
\boldsymbol{S}_{\mathrm{tra} 2 p}=\left(\begin{array}{cccc}
0 & 0 & \cdots & 0 \\
0 & 0 & \cdots & 0 \\
k_{F 1} & k_{F 2} & \cdots & k_{F 2 p}
\end{array}\right), \\
\boldsymbol{u}_{2 p}=\sum_{i=1}^{2 p} \omega_{M i}^{2} \boldsymbol{\varepsilon}_{M i},
\end{gathered}
$$

where $\operatorname{span}\left\{\varepsilon_{M 1}, \varepsilon_{M 2}, \ldots, \varepsilon_{M 2 p}\right\}$ are the basis vectors of a right-handed $2 p$-dimensional real vector space $\mathbb{R}^{2 p}$. 


\subsection{State equations, maneuvers, and flight states (or flight operating points) of multirotors}

Dynamical system state equations of rotations for each multirotor with the symmetrical standard configurations $(p=2,3,4)$ can be written as follows in explicit forms in terms of Euler angle state variables $(\boldsymbol{x}, \dot{\boldsymbol{x}})^{\mathrm{T}} \in \Sigma_{\text {rot }} \in \mathbb{R}^{6}$ and input vector functions of time $\boldsymbol{u}_{2 p} \in \Lambda \subset \mathbb{R}^{2 p}$ that control the outer generalized forces acting on the multirotor (as shown in Appendix 1):

$$
\begin{gathered}
\frac{d}{d t}\left(\begin{array}{c}
\boldsymbol{x} \\
\dot{\boldsymbol{x}}
\end{array}\right)=\left(\begin{array}{c}
\dot{\boldsymbol{x}} \\
\boldsymbol{Y}(\boldsymbol{\eta}, \dot{\boldsymbol{x}})+\boldsymbol{Z}(\boldsymbol{\eta}) \boldsymbol{S}_{\mathrm{rot} 2 p} \boldsymbol{u}_{2 p}
\end{array}\right) \\
\boldsymbol{Z}(\boldsymbol{\eta})=\left(\boldsymbol{B}(\boldsymbol{x}) \hat{\boldsymbol{I}} \boldsymbol{B}(\boldsymbol{x})^{\mathrm{T}} \cdot \omega_{\dot{\boldsymbol{x}}}(\psi, \theta)\right)^{-1} \cdot \boldsymbol{B}(\boldsymbol{x}) \\
\boldsymbol{Y}(\boldsymbol{\eta}, \dot{\boldsymbol{x}})=-\left(\boldsymbol{B}(\boldsymbol{x}) \hat{\boldsymbol{I}} \boldsymbol{B}(\boldsymbol{x})^{\mathrm{T}} \cdot \omega_{\dot{\boldsymbol{x}}}(\psi, \theta)\right)^{-1}\left(\dot{\boldsymbol{B}}(\boldsymbol{x}, \dot{\boldsymbol{x}}) \hat{\boldsymbol{I}} \boldsymbol{B}(\boldsymbol{x})^{\mathrm{T}} \cdot \omega_{\dot{\boldsymbol{x}}}(\psi, \theta)+\boldsymbol{B}(\boldsymbol{x}) \hat{\boldsymbol{I}} \boldsymbol{B}(\boldsymbol{x})^{\mathrm{T}} \cdot \dot{\omega}_{\dot{\boldsymbol{x}}}(\psi, \theta, \dot{\psi}, \dot{\theta})\right) \cdot \dot{\boldsymbol{x}},
\end{gathered}
$$

where $\boldsymbol{x}=(\psi, \theta, \phi)^{\mathrm{T}}=(\psi, \boldsymbol{\eta})^{\mathrm{T}}, \boldsymbol{\eta}=(\theta, \phi)^{\mathrm{T}}, \dot{\boldsymbol{x}}=(\dot{\psi}, \dot{\theta}, \dot{\phi})^{\mathrm{T}}=(\dot{\psi}, \dot{\boldsymbol{\eta}})^{\mathrm{T}}, \dot{\boldsymbol{\eta}}=(\dot{\theta}, \dot{\phi})^{\mathrm{T}}$, and $\rho \in \mathbb{N}$. $\boldsymbol{\omega}=\boldsymbol{\omega}_{\dot{\boldsymbol{x}}} \dot{\boldsymbol{x}} ; \boldsymbol{\omega}_{\dot{\boldsymbol{x}}}$ denotes the partial derivative of $\boldsymbol{\omega}$ with respect to $\dot{\boldsymbol{x}}$.

L e t $(\boldsymbol{x}(t), \dot{\boldsymbol{x}}(t))^{\mathrm{T}}=\left(\boldsymbol{\phi}_{1}\left(t,\left(\boldsymbol{x}_{0}, \dot{\boldsymbol{x}}_{0}\right)^{\mathrm{T}}, \boldsymbol{u}\right), \boldsymbol{\phi}_{2}\left(t,\left(\boldsymbol{x}_{0}, \dot{\boldsymbol{x}}_{0}\right)^{\mathrm{T}}, \boldsymbol{u}\right)\right)^{\mathrm{T}}=\boldsymbol{\phi}\left(t,\left(\boldsymbol{x}_{0}, \dot{\boldsymbol{x}}_{0}\right)^{\mathrm{T}}, \boldsymbol{u}\right) \in \Sigma_{\text {rot }} \subset \mathbb{R}^{6}$ with initial points $\left(t_{0},\left(\boldsymbol{x}_{0}, \dot{\boldsymbol{x}}_{0}\right)^{\mathrm{T}}\right) \in \mathbb{R} \times \Sigma_{\text {rot }} \subset \mathbb{R} \times \mathbb{R}^{6}$ and $\boldsymbol{u} \in \Lambda$ be the solution of Eq. (12). The dynamical system state equations of translations for the multirotors with the symmetrical standard configurations ( $p=2,3,4)$ are also written as follows in an explicit form in terms of translational state variables $(\boldsymbol{r}, \dot{\boldsymbol{r}})^{\mathrm{T}} \in \Sigma_{\text {tra }} \subset \mathbb{R}^{6}$ and input vector functions of time $\boldsymbol{u}_{2 p} \in \Lambda \subset \mathbb{R}^{2 p}$ (as shown in Appendix 1):

$$
\frac{d}{d t}\left(\begin{array}{c}
\boldsymbol{r} \\
\dot{\boldsymbol{r}}
\end{array}\right)=\left(\begin{array}{c}
\dot{\boldsymbol{r}} \\
-g \boldsymbol{e}_{3}+\frac{1}{m} \boldsymbol{B}\left(\phi_{1}\left(t,\left(\boldsymbol{x}_{0}, \dot{\boldsymbol{x}}_{0}\right)^{\mathrm{T}}, \boldsymbol{u}\right)\right) \boldsymbol{S}_{\mathrm{tra} 2 p} \boldsymbol{u}_{2 p}
\end{array}\right),
$$

where $m$ is the total mass of the multirotor and $g$ is the gravitational acceleration.

Here, by using the state variables of rotational motion $\boldsymbol{x}=(\psi, \theta, \phi)^{\mathrm{T}}, \dot{\boldsymbol{x}}=(\dot{\psi}, \dot{\theta}, \dot{\phi})^{\mathrm{T}}$ and translational motion $\boldsymbol{r}=\left(r_{1}, r_{2}, r_{3}\right)^{\mathrm{T}}, \dot{\boldsymbol{r}}=\left(\dot{r}_{1}, \dot{r}_{2}, \dot{r}_{3}\right)^{\mathrm{T}}$ (Fig. 5), we represent the multirotor maneuvers and flight states in many applications as shown in Table 2 . The multirotor uses $2 p$ fixed motors for flight. The angular velocities of the motors $\boldsymbol{u}_{2 p}$ are directly used to achieve the flight states in Table 2. In the case that the motion of yaw $\psi$, pitch $\theta$, and roll $\phi$ is fixed, from Eq. (12), $d / d t(\boldsymbol{x}, \dot{\boldsymbol{x}})^{\mathrm{T}}=\left(\mathbf{0}_{3}, \mathbf{0}_{3}\right)^{\mathrm{T}}$ and $\mathbf{0}_{3}=(0,0,0)^{\mathrm{T}}$ hold. Then, as shown in Eq. (15), since $\ddot{\boldsymbol{r}}$ depends on $\boldsymbol{x}(t)=\phi_{1}\left(t,\left(\boldsymbol{x}_{0}, \dot{\boldsymbol{x}}_{0}\right)^{\mathrm{T}}, \boldsymbol{u}\right)$, the motion of $r_{i}$ is given by $d / d t \dot{r}_{i}=\left\langle\boldsymbol{e}_{i}, 1 / m \boldsymbol{B}(\boldsymbol{x}) \boldsymbol{S}_{\operatorname{tra} 2 p} \boldsymbol{u}_{2 p}\right\rangle, i=1,2$. The motion of $r_{3}$ such that multirotors always maintain or control their altitudes is given by $d / d t \dot{r}_{3}=\left\langle\boldsymbol{e}_{3},-g \boldsymbol{e}_{3}+1 / m \boldsymbol{B}(x) \boldsymbol{S}_{\operatorname{tra} 2 p} \boldsymbol{u}_{2 p}\right\rangle$.

The operating and equilibrium points of multirotors can be related to the flight states in Table 2. In the following, we give Definition 1 for the operating and equilibrium points of multirotors. 


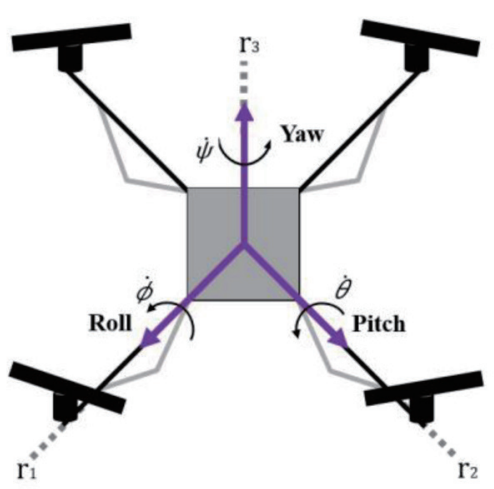

Fig. 5. (Color online) Rotational and translational motions of the quadrotor (one of the multirotors).

Table 2

Multirotor maneuvers and flight states of Fig. 5.

\begin{tabular}{|c|c|c|}
\hline Maneuver & Related state variables & Flight state \\
\hline $\begin{array}{l}\text { (i-1) Altitude up } \\
\text { control }\end{array}$ & $r_{3}$ direction, $d / d t \dot{r}_{3}>0$ & $\begin{array}{l}\text { Lifting the multirotor under gravity and } \\
\text { moving it up }\end{array}$ \\
\hline $\begin{array}{l}\text { (i-2) Altitude down } \\
\text { control }\end{array}$ & $r_{3}$ direction, $d / d t \dot{r}_{3}<0$ & $\begin{array}{l}\text { Lifting the multirotor under gravity and } \\
\text { moving it down }\end{array}$ \\
\hline (ii) Hover control & $\begin{array}{c}r_{3} \text { direction, } \dot{r}_{1}=\dot{r}_{2}=\dot{r}_{3}=0 \\
d / d t \dot{r}_{1}=d / d t \dot{r}_{2}=d / d t \dot{r}_{3}=0, \dot{\psi}=\dot{\theta}=\dot{\phi}=0\end{array}$ & $\begin{array}{l}\text { Being in equilibrium by lifting it under } \\
\text { gravity, no rotational motion, and remaining } \\
\text { in one place in the air }\end{array}$ \\
\hline $\begin{array}{l}\text { (iii-1) Yaw-changing } \\
\text { forward control }\end{array}$ & $\psi, 0 \leq \psi<2 \pi, \dot{\psi}>0$ & Yaw changing by counterclockwise turning \\
\hline $\begin{array}{l}\text { (iii-2) Yaw-changing } \\
\text { backward control }\end{array}$ & $\psi,-2 \pi<\psi \leq 0, \dot{\psi}<0$ & Yaw changing by clockwise turning \\
\hline $\begin{array}{l}\text { (iv-1) Pitch-changing } \\
\text { forward control }\end{array}$ & $\begin{array}{c}r_{1} \text { direction, } d / d t \dot{r}_{1}>0 \\
\theta, 0 \leq \theta<\pi / 2, \dot{\theta}>0\end{array}$ & $\begin{array}{l}\text { Pitch turning counterclockwise, nose } \\
\text { dropping down, tail lifting up, and } \\
\text { beginning to accelerate forward }\end{array}$ \\
\hline $\begin{array}{l}\text { (iv-2) Pitch-changing } \\
\text { backward control }\end{array}$ & $\begin{array}{c}r_{1} \text { direction, } d / d t \dot{r}_{1}<0 \\
\theta,-\pi / 2<\theta \leq 0, \dot{\theta}<0\end{array}$ & $\begin{array}{l}\text { Pitch turning clockwise, nose lifting up, tail } \\
\text { dropping down, and beginning to accelerate } \\
\text { backward }\end{array}$ \\
\hline $\begin{array}{l}\text { (v-1) Roll-changing } \\
\text { forward control }\end{array}$ & $\begin{array}{c}r_{2} \text { direction, } d / d t \dot{r}_{2}>0 \\
\phi,-\pi / 2<\phi \leq 0, \dot{\phi}<0\end{array}$ & $\begin{array}{l}\text { Roll turning clockwise, right side lifting up, } \\
\text { left side dropping down, and beginning to } \\
\text { accelerate sideways to the left }\end{array}$ \\
\hline $\begin{array}{l}\text { (v-2) Roll-changing } \\
\text { backward control }\end{array}$ & $\begin{array}{c}r_{2} \text { direction, } d / d t \dot{r}_{2}<0 \\
\phi, 0 \leq \phi<\pi / 2, \quad \dot{\phi}>0\end{array}$ & $\begin{array}{l}\text { Roll turning counterclockwise, right side } \\
\text { dropping down, left side lifting up, and } \\
\text { beginning to accelerate sideways to the } \\
\text { right }\end{array}$ \\
\hline
\end{tabular}

Definition 1: Operating points (hereinafter, referred to as "flight operating points") of multirotors $\left(\boldsymbol{x}_{\mathrm{op}}, \dot{\boldsymbol{x}}_{\mathrm{op}}\right)^{\mathrm{T}} \in \mathbb{R}^{3} \times \mathbb{R}^{3}, \boldsymbol{x}_{\mathrm{op}}=\left(\psi_{\mathrm{op}}, \boldsymbol{\eta}_{\mathrm{op}}\right)^{\mathrm{T}}, \boldsymbol{\eta}_{\mathrm{op}}=\left(\theta_{\mathrm{op}}, \phi_{\mathrm{op}}\right)^{\mathrm{T}}$ and $\left(\boldsymbol{r}_{\mathrm{op}}, \dot{\boldsymbol{r}}_{\mathrm{op}}\right)^{\mathrm{T}} \in \mathbb{R}^{3} \times \mathbb{R}^{3}$ are determined as

$$
\left(\begin{array}{c}
\dot{\boldsymbol{x}}_{\mathrm{op}} \\
\boldsymbol{Z}\left(\boldsymbol{\eta}_{\mathrm{op}}\right) \boldsymbol{S}_{\mathrm{rot} 2 p} \boldsymbol{u}_{2 p(\mathrm{op})}
\end{array}\right)=\left(\begin{array}{c}
\mathbf{0}_{3} \\
\mathbf{0}_{3}
\end{array}\right),
$$




$$
\begin{gathered}
\left(\begin{array}{c}
\dot{r}_{3(\mathrm{op})} \\
\left\langle\boldsymbol{e}_{3},-g \boldsymbol{e}_{3}+\frac{1}{m} \boldsymbol{B}\left(\boldsymbol{x}_{\mathrm{op}}\right) \boldsymbol{S}_{\mathrm{tra} 2 p} \boldsymbol{u}_{2 p(\mathrm{op})}\right\rangle
\end{array}\right)=\left(\begin{array}{c}
0 \\
c
\end{array}\right), \\
\boldsymbol{Z}\left(\boldsymbol{\eta}_{\mathrm{op}}\right)=\left(\boldsymbol{B}\left(\boldsymbol{x}_{\mathrm{op}}\right) \hat{\boldsymbol{I}} \boldsymbol{B}\left(\boldsymbol{x}_{\mathrm{op}}\right)^{\mathrm{T}} \cdot \omega_{\dot{\boldsymbol{x}}}\left(\psi_{\mathrm{op}}, \theta_{\mathrm{op}}\right)\right)^{-1} \cdot \boldsymbol{B}\left(\boldsymbol{x}_{\mathrm{op}}\right), \\
\ddot{r}_{1}=\left\langle\boldsymbol{e}_{1}, \frac{1}{m} \boldsymbol{B}\left(\boldsymbol{x}_{\mathrm{op}}\right) \boldsymbol{S}_{\mathrm{tra} 2 p} \boldsymbol{u}_{2 p(\mathrm{op})}\right\rangle, \\
\ddot{\boldsymbol{r}}_{2}=\left\langle\boldsymbol{e}_{2}, \frac{1}{m} \boldsymbol{B}\left(\boldsymbol{x}_{\mathrm{op}}\right) \boldsymbol{S}_{\mathrm{tra} 2 p} \boldsymbol{u}_{2 p(\mathrm{op})}\right\rangle, \\
\ddot{\boldsymbol{r}}_{3}=c,
\end{gathered}
$$

where $c \in \mathbb{R}$ is a constant and $\mathbf{0}_{3}=(0,0,0)^{\mathrm{T}}$. If $c=0$, the flight operating points $\left(\boldsymbol{x}_{\mathrm{op}}, \dot{\boldsymbol{x}}_{\mathrm{op}}\right)^{\mathrm{T}}$ and $\left(\boldsymbol{r}_{\mathrm{op}}, \dot{\boldsymbol{r}}_{\mathrm{op}}\right)^{\mathrm{T}}$ have a constant altitude. Furthermore, if $\dot{r}_{1}=\dot{r}_{2}=\ddot{r}_{1}=\ddot{r}_{2}=0$ and $c=0$ (i.e., $\ddot{r}_{3}=0$ ), the flight operating points are also identified as equilibrium points $\left(\boldsymbol{x}_{e}, \dot{\boldsymbol{x}}_{e}\right)^{\mathrm{T}}$ and $\left(\boldsymbol{r}_{e}, \dot{\boldsymbol{r}}_{e}\right)^{\mathrm{T}}$ and occur in hovering flight.

\section{Problem of Multirotor Flight States to Avoid a Crash in the Case of Complete Propeller Motor Failures}

In this section, in the case of complete propeller motor failures, we consider a method of providing motor speed control signals to achieve flight states to avoid a crash based on the state variable approach.

First, we assume that the motors in the $i_{1}$ th, $i_{2}$ th, $\ldots, i_{n}$ th rotors $\left(1 \leq i_{1}<i_{2}<\cdots<i_{n} \leq 2 p\right.$, $1 \leq n \leq 2 p-2, p=2,3,4, n \in \mathbb{N}$ ) have completely failed. Then, the motor speed control signal vector of the remaining motors is determined by

$$
\boldsymbol{u}_{2 p-n}^{i_{1}, i_{2}, \ldots, i_{n}}=\sum_{j=1}^{i_{1}-1} \omega_{M j}^{2} \boldsymbol{\varepsilon}_{M j}+\sum_{j=i_{1}+1}^{i_{2}-1} \omega_{M j}^{2} \boldsymbol{\varepsilon}_{M j}+\cdots+\sum_{j=i_{n}+1}^{2 p} \omega_{M j}^{2} \boldsymbol{\varepsilon}_{M j}
$$

The remaining moments of $\boldsymbol{S}_{\operatorname{rot} 2 p} \boldsymbol{u}_{2 p-n}^{i_{1}, i_{2}, \ldots, i_{n}}$ and the remaining translational forces of $\boldsymbol{S}_{\text {tra } 2 p} \boldsymbol{u}_{2 p-n}^{i_{1}, i_{2}, \ldots, i_{n}}, p=2,3,4$, are respectively given as

$$
\begin{gathered}
\boldsymbol{S}_{\mathrm{rot} 2 p} \boldsymbol{u}_{2 p-n}^{i_{1}, i_{2}, \ldots, i_{n}}=\boldsymbol{S}_{\mathrm{rot} 2 p-n}^{i_{1}, i_{2}, \ldots, i_{n}} \boldsymbol{u}_{2 p-n}^{i_{1}, i_{2}, \ldots, i_{n}}, \\
\boldsymbol{S}_{\mathrm{tra} 2 p} \boldsymbol{u}_{2 p-n}^{i_{1}, i_{2}, \ldots, i_{n}}=\boldsymbol{S}_{\text {tra2 } p-n}^{i_{1}, i_{2}, \ldots, i_{n}} \boldsymbol{u}_{2 p-n}^{i_{1}, i_{2}, \ldots, i_{n}},
\end{gathered}
$$

where $\boldsymbol{S}_{\xi 2 p-n}^{i_{1}, i_{2}, \ldots, i_{n}}$ is the matrix $\boldsymbol{S}_{\xi 2 p}$ with the $i_{1}$ th, $i_{2}$ th, .., $i_{n}$ th rows deleted ( $\xi=$ rot, tra).

Example 1: When the motors of the 1st, 3rd, and 5th rotors of the hexarotor $(p=3)$ have 
completely failed (in Fig. 6), the motor speed control signal vector of the remaining motors is determined as

$$
\boldsymbol{u}_{2 p-n}^{i_{1}, i_{2}, \ldots, i_{n}}=\boldsymbol{u}_{3}^{1,3,5}=\omega_{M 2}^{2} \boldsymbol{\varepsilon}_{M 2}+\omega_{M 4}^{2} \boldsymbol{\varepsilon}_{M 4}+\omega_{M 6}^{2} \boldsymbol{\varepsilon}_{M 6},
$$

where $i_{1}=1, i_{2}=3, i_{3}=5$, and $n=3 . \boldsymbol{S}_{\text {rot3 }} \boldsymbol{u}_{3}^{1,3,5}$ and $\boldsymbol{S}_{\text {tra } 3} \boldsymbol{u}_{3}^{1,3,5}$ are respectively given as

$$
\begin{gathered}
\boldsymbol{S}_{\mathrm{rot} 3} \boldsymbol{u}_{3}^{1,3,5}=\boldsymbol{S}_{\mathrm{rot} 3}^{1,3,5} \boldsymbol{u}_{3}^{1,3,5}, \\
\boldsymbol{S}_{\text {tra3 }} \boldsymbol{u}_{3}^{1,3,5}=\boldsymbol{S}_{\mathrm{tra3}}^{1,3,5} \boldsymbol{u}_{3}^{1,3,5}, \\
\boldsymbol{S}_{\mathrm{rot} 3}^{1,3,5} \boldsymbol{u}_{3}^{1,3,5}=\left(\begin{array}{ccc}
-\frac{\sqrt{3}}{2} \ell \cdot k_{F 2} & 0 & \frac{\sqrt{3}}{2} \ell \cdot k_{F 6} \\
0.5 \ell \cdot k_{F 2} & -\ell \cdot k_{F 4} & 0.5 \ell \cdot k_{F 6} \\
k_{M 2} & k_{M 4} & k_{M 6}
\end{array}\right)\left(\begin{array}{c}
\omega_{M 2}^{2} \\
\omega_{M 4}^{2} \\
\omega_{M 6}^{2}
\end{array}\right), \\
\boldsymbol{S}_{\text {tra3 }}^{1,3,5} \boldsymbol{u}_{3}^{1,3,5}=\left(\begin{array}{ccc}
0 & 0 & 0 \\
0 & 0 & 0 \\
k_{F 2} & k_{F 4} & k_{F 6}
\end{array}\right)\left(\begin{array}{c}
\omega_{M 2}^{2} \\
\omega_{M 4}^{2} \\
\omega_{M 6}^{2}
\end{array}\right),
\end{gathered}
$$

where $\boldsymbol{u}_{3}^{1,3,5} \in \mathbb{R}^{3} . \boldsymbol{S}_{\xi 3}^{1,3,5}$ is the matrix $\boldsymbol{S}_{\xi 6}$ with the 1st, 3rd, and 5th rows deleted ( $\xi=$ rot, tra).

Note that in the case of the hexarotor $(p=3)$, the direction of rotation of the motors on each diagonal line is not the same (Fig. 3). Therefore, the case of four motors of the hexarotor having completely failed $(n=4)$ is not treated $\left(1 \leq i_{1}<i_{2}<\cdots<i_{n} \leq 2 p, 1 \leq n \leq 2 p-3, p=3, n \in \mathbb{N}\right)$.

Here, we summarize the state equations of the multirotors with the symmetrical standard configurations $(p=2,3,4)$ in the case of complete propeller motor failures using the Euler angle rotational equation [Eq. (12)] and the translational equation [Eq. (15)] as follows:

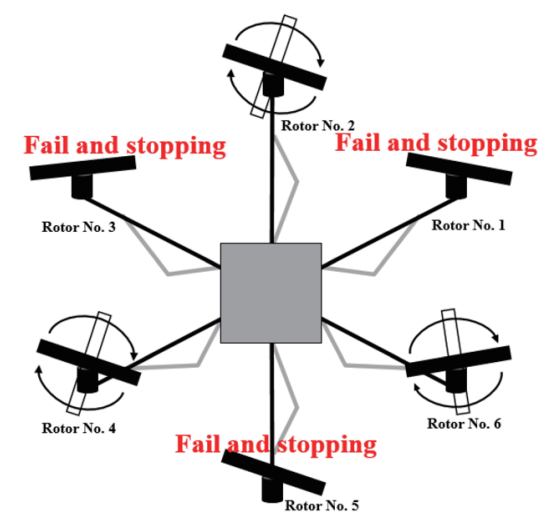

Fig. 6. (Color online) Example of a hexarotor flight when the 1st, 3rd, and 5th rotors have completely failed. 


$$
\begin{aligned}
& \frac{d}{d t}\left(\begin{array}{c}
\boldsymbol{x} \\
\dot{\boldsymbol{x}}
\end{array}\right)=\left(\begin{array}{c}
\dot{\boldsymbol{x}} \\
\boldsymbol{Y}(\boldsymbol{\eta}, \dot{\boldsymbol{x}})+\boldsymbol{Z}(\boldsymbol{\eta}) \boldsymbol{S}_{\operatorname{rot} 2 p-n}^{i_{1}, i_{2}, \ldots, i_{n}} \boldsymbol{u}_{2 p-n}^{i_{1}, i_{2}, \ldots, i_{n}}
\end{array}\right), \\
& \frac{d}{d t}\left(\begin{array}{c}
\boldsymbol{r} \\
\dot{\boldsymbol{r}}
\end{array}\right)=\left(\begin{array}{c}
\dot{\boldsymbol{r}} \\
-g \boldsymbol{e}_{3}+\frac{1}{m} \boldsymbol{B}(\boldsymbol{x}) \boldsymbol{S}_{\operatorname{tra} 2 p-n}^{i_{1}, i_{2}, \ldots, i_{n}} \boldsymbol{u}_{2 p-n}^{i_{1}, i_{2}, \ldots, i_{n}}
\end{array}\right) .
\end{aligned}
$$

In the case of complete propeller motor failures, the flight operating and equilibrium points of multirotors can also be related to the two types of multirotor flight state in Table 3 to avoid a crash when some motors fail and stop. In the following, we give Definition 2 for the flight operating and equilibrium points of multirotors to achieve the flight states in Table 3 to avoid a crash when some motors fail and stop. We propose Method 1 based on Definition 2 to provide motor speed control signals to achieve the flight states in Table 3 to avoid a crash.

We assume two types of multirotor flight states to avoid a crash when some motors fail and stop.

Definition 2: When some motors fail and stop, the flight operating points of multirotors to achieve the flight states in Table $3\left(\boldsymbol{x}_{\mathrm{op}}, \dot{\boldsymbol{x}}_{\mathrm{op}}\right)^{\mathrm{T}} \in \mathbb{R}^{3} \times \mathbb{R}^{3}, \boldsymbol{x}_{\mathrm{op}}=\left(\psi_{\mathrm{op}}, \boldsymbol{\eta}_{\mathrm{op}}\right)^{\mathrm{T}}, \boldsymbol{\eta}_{\mathrm{op}}=\left(\theta_{\mathrm{op}}, \phi_{\mathrm{op}}\right)^{\mathrm{T}}$, and $\left(\boldsymbol{r}_{\mathrm{op}}, \dot{\boldsymbol{r}}_{\mathrm{op}}\right)^{\mathrm{T}} \in \mathbb{R}^{3} \times \mathbb{R}^{3}$ to avoid a crash are determined as

$$
\begin{gathered}
\left(\begin{array}{c}
\dot{\boldsymbol{x}}_{\mathrm{op}} \\
\boldsymbol{Z}\left(\boldsymbol{\eta}_{\mathrm{op}}\right) \boldsymbol{S}_{\mathrm{rot} 2 p-n}^{i_{1}, i_{2}, \ldots, i_{n}} \boldsymbol{u}_{2 p-n(\mathrm{op})}^{i_{1}, i_{2}, \ldots, i_{n}}
\end{array}\right)=\left(\begin{array}{c}
\mathbf{0}_{3} \\
\mathbf{0}_{3}
\end{array}\right), \\
\left(\begin{array}{c}
\dot{r}_{3(\mathrm{op})} \\
\left\langle\boldsymbol{e}_{3},-g \boldsymbol{e}_{3}+\frac{1}{m} \boldsymbol{B}\left(\boldsymbol{x}_{\mathrm{op}}\right) \boldsymbol{S}_{\mathrm{tra} 2 p-n}^{i_{1}, i_{2}, \ldots, i_{n}} \boldsymbol{u}_{2 p-n(\mathrm{op})}^{i_{1}, i_{2}, \ldots, i_{n}}\right\rangle
\end{array}\right)=\left(\begin{array}{c}
0 \\
c
\end{array}\right), \\
\boldsymbol{Z}\left(\boldsymbol{\eta}_{\mathrm{op}}\right)=\left(\boldsymbol{B}\left(\boldsymbol{x}_{\mathrm{op}}\right) \hat{\boldsymbol{I}} \boldsymbol{B}\left(\boldsymbol{x}_{\mathrm{op}}\right)^{\mathrm{T}} \cdot \boldsymbol{\omega}_{\dot{\boldsymbol{x}}}\left(\psi_{\mathrm{op}}, \theta_{\mathrm{op}}\right)\right)^{-1} \cdot \boldsymbol{B}\left(\boldsymbol{x}_{\mathrm{op}}\right), \\
\ddot{\boldsymbol{r}}_{1}=\left\langle\boldsymbol{e}_{1}, \frac{1}{m} \boldsymbol{B}\left(\boldsymbol{x}_{\mathrm{op}}\right) \boldsymbol{S}_{\mathrm{tra} 2 p-n}^{i_{1}, i_{2}, \ldots, i_{n}} \boldsymbol{u}_{2 p-n(\mathrm{op})}^{i_{1}, i_{2}, \ldots, i_{n}}\right\rangle, \\
\ddot{r}_{2}=\left\langle\boldsymbol{e}_{2}, \frac{1}{m} \boldsymbol{B}\left(\boldsymbol{x}_{\mathrm{op}}\right) \boldsymbol{S}_{\mathrm{tra} 2 p-n}^{i_{1}, i_{2}, \ldots, i_{n}} \boldsymbol{u}_{2 p-n(\mathrm{op})}^{i_{1}, i_{2}, \ldots, i_{n}}\right\rangle,
\end{gathered}
$$

Table 3

Two types of multirotor flight states to avoid a crash.

\begin{tabular}{lc}
\hline Type & Achieved flight states in Table 2 \\
\hline (I) No problem & All (e.g., in Fig. 7). \\
(II) Admissible problem & Yaw angles cannot be controlled. Yaw angles are freely changing (e.g., in Fig. 8).
\end{tabular}

Type (I): Among the remaining motors, some must be stopped to achieve all states in Table 2 when certain motors fail and stop.

Type (II): Among the remaining motors, some must be stopped to achieve all states (except for yaw control) in Table 2 when certain motors fail and stop.

Types (I) and (II) represent the severity levels of complete propeller motor failure. Types (I) and (II) are determined by the equation forms of Eq. (41) in Method 1. Type (II) is more severe than type (I) because yaw angles cannot be controlled. 


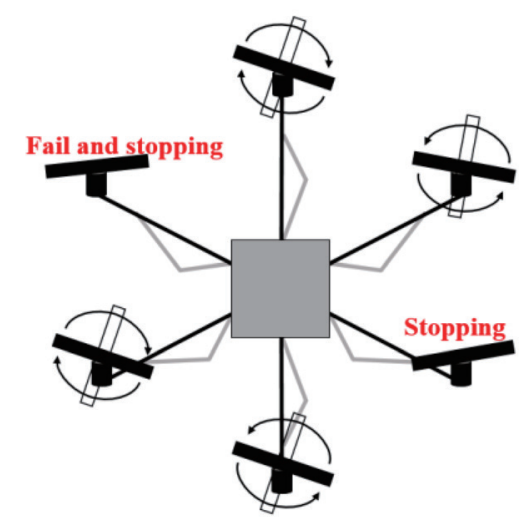

Fig. 7. (Color online) Example of type (I) no problem where one of the remaining motors must be stopped to achieve all the states of the hexarotor in Table 2 when one motor fails and stops.

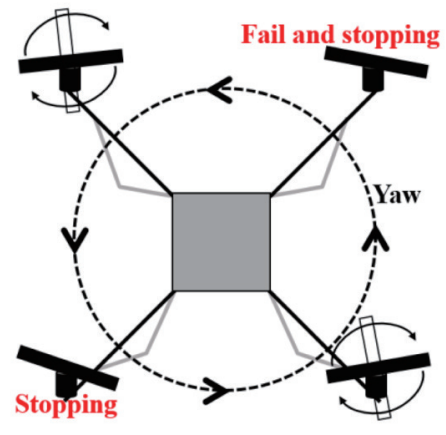

Fig. 8. (Color online) Example of type (II) admissible problem where one of the remaining motors must be stopped to achieve all states (except for yaw control) of the quadrotor in Table 2 when one motor fails and stops.

$$
\ddot{r}_{3}=c,
$$

where $c \in \mathbb{R}$ is a constant and $\mathbf{0}_{3}=(0,0,0,)^{\mathrm{T}}$. If $c=0$, the flight operating points $\left(\boldsymbol{x}_{\mathrm{op}}, \dot{\boldsymbol{x}}_{\mathrm{op}}\right)^{\mathrm{T}}$ and $\left(\boldsymbol{r}_{\mathrm{op}}, \dot{\boldsymbol{r}}_{\mathrm{op}}\right)^{\mathrm{T}}$ have a constant altitude. Furthermore, if $\dot{r}_{1}=\dot{r}_{2}=\ddot{r}_{1}=\ddot{r}_{2}=0$ and $c=0$ (i.e., $\ddot{r}_{3}=0$ ), the flight operating points are called equilibrium points $\left(\boldsymbol{x}_{\mathrm{e}}, \dot{\boldsymbol{x}}_{\mathrm{e}}\right)^{\mathrm{T}}$ and $\left(\boldsymbol{r}_{\mathrm{e}}, \dot{\boldsymbol{r}}_{\mathrm{e}}\right)^{\mathrm{T}}$ and occur in hovering flights. In the case of type (II) in Table 3, the first and fourth lines of Eq. (32) are automatically determined. Therefore, Eq. (32) is rewritten as

$$
\begin{gathered}
\left(\begin{array}{c}
\dot{\boldsymbol{\eta}}_{\mathrm{op}} \\
\left\langle\boldsymbol{\varepsilon}_{2}, \boldsymbol{Z}\left(\boldsymbol{\eta}_{\mathrm{op}}\right) \boldsymbol{S}_{\mathrm{rot}, i_{2}, \ldots, i_{n}, i_{n}} \boldsymbol{u}_{2, i_{2}, \ldots, i_{n}}^{i_{2}-n\left(\mathrm{o}_{2}\right)}\right. \\
\left\langle\boldsymbol{\varepsilon}_{3}, \boldsymbol{Z}\left(\boldsymbol{\eta}_{\mathrm{op}}\right) \boldsymbol{S}_{\mathrm{rot} 2 p-n}^{i_{1}, i_{2}, \ldots, i_{n}} \boldsymbol{u}_{2 p-n(\mathrm{op})}^{i_{1}, i_{2}, \ldots, l_{n}}\right.
\end{array}\right)=\left(\begin{array}{r}
\mathbf{0}_{2} \\
0 \\
0
\end{array}\right), \\
\ddot{\psi}=\left\langle\boldsymbol{\varepsilon}_{1}, \boldsymbol{Y}(\boldsymbol{\eta}, \dot{\boldsymbol{x}})+\boldsymbol{Z}(\boldsymbol{\eta}) \boldsymbol{S}_{\mathrm{rot} 2 p-n}^{i_{1}, i_{2}, \ldots, i_{n}} \boldsymbol{u}_{2 p-n}^{i_{1}, i_{2}, \ldots, i_{n}}\right\rangle, \\
\dot{\psi}=c_{\psi} t+\dot{\psi}(0),
\end{gathered}
$$

where $c_{\psi} \in \mathbb{R}$ is a constant. Note that maneuver (ii) in Table 2, hover control, cannot be achieved.

Method 1: Let $\boldsymbol{F}\left(\boldsymbol{x}, \boldsymbol{u}_{2 p-n}^{i_{1}, i_{2}, \ldots, i_{n}}\right)$ be the following $C^{1}$ function taking values in $\mathbb{R}^{2 p-n}, p=2,3,4$ with a neighborhood of Euler angle state variables $\tilde{\boldsymbol{x}}$ in $\mathbb{R}^{3}$ and the motor speed control signal vector of the remaining motors $\tilde{\boldsymbol{u}}_{2 p-n}^{i_{1}, i_{2}, \ldots, i_{n}}$ in $\mathbb{R}^{2 p-n}$ with $\boldsymbol{F}\left(\tilde{\boldsymbol{x}}, \tilde{\boldsymbol{u}}_{2 p-n}^{i_{1}, i_{2}, \ldots, i_{n}}\right)=\mathbf{0}_{2 p-n}=$ $(0,0, \ldots, 0)^{\mathrm{T}}$, including both lower sections of $\boldsymbol{Z}\left(\boldsymbol{\eta}_{\mathrm{op}}\right) \boldsymbol{S}_{\mathrm{rot} 2 p-n}^{i_{1}, i_{2}, \ldots, i_{n}} \boldsymbol{u}_{2 p-n(\mathrm{op})}^{i_{1}, i_{2}, \ldots, i_{n}}$ in Eq. (32) and 
$\left\langle\boldsymbol{e}_{3},-g \boldsymbol{e}_{3}+1 / m \boldsymbol{B}\left(\boldsymbol{x}_{\mathrm{op}}\right) \boldsymbol{S}_{\text {tra2 } 2-n}^{i_{1}, i_{2}, \ldots, i_{n}} \boldsymbol{u}_{2 p-n(\mathrm{op})}^{i_{1}, i_{2}, \ldots, i_{n}}\right\rangle$ in Eq. (33):

$$
\boldsymbol{F}\left(\boldsymbol{x}, \boldsymbol{u}_{2 p-n}^{i_{1}, i_{2}, \ldots, i_{n}}\right)=\boldsymbol{A}_{2 p-n}^{i_{1}, i_{2}, \ldots, i_{n}}(\boldsymbol{x}) \boldsymbol{u}_{2 p-n}^{i_{1}, i_{2}, \ldots, i_{n}}-\boldsymbol{b}_{2 p-n} .
$$

In the case of $p=2$,

$$
\boldsymbol{A}_{4-n}^{i_{1}, i_{2}, \ldots, i_{n}}(\boldsymbol{\eta}) \in \mathbb{R}^{(4-n) \times(4-n)}=\left(\begin{array}{c}
\boldsymbol{Z}(\boldsymbol{\eta}) \boldsymbol{S}_{\mathrm{rot}}^{i_{1}, i_{2}, \ldots, i_{n}} \\
\boldsymbol{e}_{3}^{\mathrm{T}} \frac{1}{m} \boldsymbol{B}(\boldsymbol{x}) \boldsymbol{S}_{\text {tra } 4, n-n}^{l_{1}, \ldots, i_{n}}
\end{array}\right),
$$

where $c \in \mathbb{R}$ is a constant and $\boldsymbol{b}_{4-n}=(\ddot{\psi}, \ddot{\theta}, \ddot{\phi}, c+g)^{\mathrm{T}} \in \mathbb{R}^{4-n}$.

In the case of $p=3$ or 4 ,

$$
\begin{aligned}
& \boldsymbol{A}_{2 p-n}^{i_{1}, i_{2}, \ldots, i_{n}}(\boldsymbol{x}) \in \mathbb{R}^{(2 p-n) \times(2 p-n)}=\left(\begin{array}{c}
\boldsymbol{A}_{4 \times(2, \ldots, n)}^{i_{1}, i_{2}, \ldots, i_{n}}(\boldsymbol{\eta}) \\
\boldsymbol{Q}_{(2 p-4) \times(2 p-n)}^{\left.i_{1}, i_{2}, \ldots, \eta_{n}\right)}
\end{array}\right), \\
& \boldsymbol{A}_{4 \times(2 p-n)}^{i_{1}, i_{2}, \ldots, i_{n}}(\boldsymbol{\eta}) \in \mathbb{R}^{4 \times(2 p-n)}=\left(\begin{array}{c}
\boldsymbol{Z}(\boldsymbol{\eta}) \boldsymbol{S}_{\operatorname{rot}}^{i_{1}, i_{2}, \ldots, i_{n}}, i_{n} \\
\boldsymbol{e}_{3}^{\mathrm{T}} \frac{1}{m} \boldsymbol{B}(\boldsymbol{x}) \boldsymbol{S}_{\mathrm{tra} 2 p-n}^{i_{1}, i_{2}, \ldots, i_{n}}
\end{array}\right),
\end{aligned}
$$

where $\boldsymbol{Q}_{(2 p-4) \times(2 p-n)}^{i_{1}, i_{2}, \ldots, i_{n}} \in \mathbb{R}^{(2 p-4) \times(2 p-n)}$ is a constant matrix, $\boldsymbol{b}_{2 p-n}=\left(\ddot{\psi}, \ddot{\theta}, \ddot{\phi}, c+g, \boldsymbol{b}_{2 p-4}^{\prime}\right)^{\mathrm{T}} \in \mathbb{R}^{2 p-n}$, and $\boldsymbol{b}_{2 p-4}^{\prime} \in \mathbb{R}^{2 p-4}$ is a constant vector.

If for arbitrary $\tilde{\boldsymbol{x}} \in \mathbb{R}^{3}, \operatorname{det}\left(A_{2 p-n}^{i_{1}, i_{2}, \ldots, i_{n}}(\tilde{\boldsymbol{x}})\right) \neq 0, \tilde{\boldsymbol{u}}_{2 p-n}^{i_{1}, i_{2}, \ldots, i_{n}} \in \mathbb{R}^{2 p-n}$, such that $\boldsymbol{F}\left(\tilde{\boldsymbol{x}}, \tilde{\boldsymbol{u}}_{2 p-n}^{i_{1}, i_{2}, \ldots, i_{n}}\right)$ $=0_{2 p-n}$, then $\tilde{\boldsymbol{u}}_{2 p-n}^{i_{1}, i_{2}, \ldots, i_{n}}=\boldsymbol{A}_{2 p-n}^{i_{1}, i_{2}, \ldots, i_{n}}(\tilde{\boldsymbol{x}})^{-1} \boldsymbol{b}_{2 p-n}$ is uniquely obtained. Then, $\ddot{\psi}, \ddot{\theta}$, and $\ddot{\phi}$ are also determined as

$$
\begin{aligned}
& \ddot{\psi}=\left\langle\boldsymbol{\varepsilon}_{1}, \boldsymbol{Y}(\boldsymbol{\eta}, \dot{\boldsymbol{x}})+\boldsymbol{Z}(\boldsymbol{\eta}) \boldsymbol{S}_{\mathrm{rot} 2 p-n}^{i_{1}, i_{2}, \ldots, i_{n}} \boldsymbol{u}_{2 p-n}^{i_{1}, i_{2}, \ldots, i_{n}}\right\rangle, \\
& \ddot{\theta}=\left\langle\boldsymbol{\varepsilon}_{2}, \boldsymbol{Y}(\boldsymbol{\eta}, \dot{\boldsymbol{x}})+\boldsymbol{Z}(\boldsymbol{\eta}) \boldsymbol{S}_{\mathrm{rot} 2 p-n}^{i_{1}, i_{2}, \ldots, i_{n}} \boldsymbol{u}_{2 p-n}^{i_{1}, i_{2}, \ldots, i_{n}}\right\rangle, \\
& \ddot{\boldsymbol{\phi}}=\left\langle\boldsymbol{\varepsilon}_{3}, \boldsymbol{Y}(\boldsymbol{\eta}, \dot{\boldsymbol{x}})+\boldsymbol{Z}(\boldsymbol{\eta}) \boldsymbol{S}_{\mathrm{rot} 2 p-n}^{i_{1}, i_{2}, \ldots, i_{n}} \boldsymbol{u}_{2 p-n}^{i_{1}, i_{2}, \ldots, i_{n}}\right\rangle .
\end{aligned}
$$

Thus, $\tilde{\boldsymbol{u}}_{2 p-n}^{i_{1}, i_{2}, \ldots, i_{n}}$ in $\mathbb{R}^{2 p-n}$ are obtained as the motor speed control signals to achieve the flight states in Table 3 to avoid a crash. As the number of failed motors $n$ increases, the rank of $\boldsymbol{S}_{\text {rot } 2 p-n}^{\dot{1}_{1}, i_{2}, \ldots, i_{n}} \in \mathbb{R}^{3 \times(2 p-n)}$ or $\boldsymbol{S}_{\text {tra2 } 2 p-n}^{i_{1}, i_{2}, \ldots, i_{n}} \in \mathbb{R}^{3 \times(2 p-n)}$ and the dimensionality of $\boldsymbol{u}_{2 p-n}^{i_{1}, i_{2}, \ldots, i_{n}} \in \mathbb{R}^{2 p-n}$ decrease. Therefore, since $\boldsymbol{A}_{2 p-n}^{i_{1}, i_{2}, \ldots, i_{n}} \in \mathbb{R}^{(2 p-n) \times(2 p-n)}$ is not a square matrix, it is necessary to remove arbitrary rows to obtain square matrices. In the case of type (II) in Table 3, the yaw angle information is considered to have been removed from the beginning. 


\section{Simulations of the Flight States for Avoiding Crashes with a Multirotor Vehicle Experiencing Motor Failures}

In this section, we present typical examples of the two types of multirotor flight states in Table 3 to avoid a crash. The following results are obtained by using Method 1 with Maple symbolic computations, MATLAB matrix calculations, and MATLAB numerical simulations with the ode45 solver. ${ }^{(16)}$ Note that regarding Eq. (31), the numerical computations in the following figures are carried out using the computation results obtained from Eq. (30) together with piecewise linear interpolations in Ref. 17 of $\psi(t), \theta(t)$, and $\phi(t)$. Each motor speed control signal used to avoid a flight crash in examples (a) to (e) is the constant vector $\boldsymbol{u}_{2 p-n}^{i_{1}, i_{2}, \ldots, i_{n}}$ obtained from Method 1. Such obtained motor speed control signals $\boldsymbol{u}_{2 p-n}^{i_{1}, i_{2}, \ldots, i_{n}}$ may be insufficient to maintain the flight operating points to avoid crashes under the influence of disturbances such as wind. However, each value of $\boldsymbol{u}_{2}^{i_{1}, i_{2}, \ldots, i_{n}}$ obtained from Method 1 is indispensable for determining the desirable flight operating point in feedback controls necessary to provide stability and avoid a crash under the influence of disturbances. The numerical parameters of a quadrotor, a hexarotor, and an octorotor are given in Tables $4-6$, respectively.

In addition, we define the constant matrices of $\boldsymbol{Q}_{(2 p-4) \times 2 p} \in \mathbb{R}^{(2 p-4) \times 2 p}, p=3$ or 4 , as follows:

Table 4

Numerical parameters of a quadrotor $(p=2)$.

\begin{tabular}{lcc}
\hline Symbol & Description & Value and unit \\
\hline$m$ & Total mass of the quadrotor & $1.656(\mathrm{~kg})$ \\
$I_{11}$ & Moment of inertia of the quadrotor & $0.01982\left(\mathrm{~kg} \cdot \mathrm{m}^{2}\right)$ \\
$I_{22}$ & Moment of inertia of the quadrotor & $0.01954\left(\mathrm{~kg} \cdot \mathrm{m}^{2}\right)$ \\
$I_{33}$ & Moment of inertia of the quadrotor & $0.03221\left(\mathrm{~kg} \cdot \mathrm{m}^{2}\right)$ \\
$g$ & Gravitational acceleration & $9.80665\left(\mathrm{~m} / \mathrm{s}^{2}\right)$ \\
$k_{F}$ & Motor force coefficient of the quadrotor & $1.79 \times 10^{-7}\left(\mathrm{~N} / \mathrm{rpm}{ }^{2}\right)$ \\
$k_{M}$ & Motor moment coefficient of the quadrotor & $\left.4.38 \times 10^{-9}(\mathrm{Nm} / \mathrm{rpm})^{2}\right)$ \\
$l$ & Distance from each rotor to geometric center of the quadrotor & $0.365(\mathrm{~m})$ \\
\hline
\end{tabular}

Table 5

Numerical parameters of a hexarotor $(p=3)$.

\begin{tabular}{lcc}
\hline Symbol & Description & Value and unit \\
\hline$m$ & Total mass of the hexarotor & $2(\mathrm{~kg})$ \\
$I_{11}$ & Moment of inertia of the hexarotor & $0.02973\left(\mathrm{~kg} \cdot \mathrm{m}^{2}\right)$ \\
$I_{22}$ & Moment of inertia of the hexarotor & $0.02931\left(\mathrm{~kg} \cdot \mathrm{m}^{2}\right)$ \\
$I_{33}$ & Moment of inertia of the hexarotor & $0.048315\left(\mathrm{~kg} \cdot \mathrm{m}^{2}\right)$ \\
$g$ & Gravitational acceleration & $9.80665\left(\mathrm{~m} / \mathrm{s}^{2}\right)$ \\
$k_{F}$ & Motor force coefficient of the hexarotor & $\left.1.79 \times 10^{-7}(\mathrm{~N} / \mathrm{rpm})^{2}\right)$ \\
$k_{M}$ & Motor moment coefficient of the hexarotor & $4.38 \times 10^{-9}\left(\mathrm{Nm} / \mathrm{rpm}{ }^{2}\right)$ \\
$l$ & Distance from each rotor to geometric center of the hexarotor & $0.365(\mathrm{~m})$ \\
\hline
\end{tabular}


Table 6

Numerical parameters of an octorotor $(p=4)$.

\begin{tabular}{lcc}
\hline Symbol & Description & Value and unit \\
\hline$m$ & Total mass of the octorotor & $3.5(\mathrm{~kg})$ \\
$I_{11}$ & Moment of inertia of the octorotor & $0.03964\left(\mathrm{~kg} \cdot \mathrm{m}^{2}\right)$ \\
$I_{22}$ & Moment of inertia of the octorotor & $0.03908\left(\mathrm{~kg} \cdot \mathrm{m}^{2}\right)$ \\
$I_{33}$ & Moment of inertia of the octorotor & $0.06442\left(\mathrm{~kg} \cdot \mathrm{m}^{2}\right)$ \\
$g$ & Gravitational acceleration & $9.80665\left(\mathrm{~m} / \mathrm{s}^{2}\right)$ \\
$k_{F}$ & Motor force coefficient of the octorotor & $1.79 \times 10^{-7}\left(\mathrm{~N} / \mathrm{rpm}{ }^{2}\right)$ \\
$k_{M}$ & Motor moment coefficient of the octorotor & $4.38 \times 10^{-9}\left(\mathrm{Nm} / \mathrm{rpm}^{2}\right)$ \\
$l$ & Distance from each rotor to geometric center of the octorotor & $0.365(\mathrm{~m})$ \\
\hline
\end{tabular}

the hexarotor $(p=3)$,

$$
\boldsymbol{Q}_{2 \times 6}=\left(\begin{array}{cccccc}
0 & 1 & -1 & 0 & 0 & 0 \\
0 & 0 & 0 & 1 & -1 & 0
\end{array}\right)
$$

the octorotor $(p=4)$,

$$
\boldsymbol{Q}_{4 \times 8}=\left(\begin{array}{cccccccc}
0 & 1 & -1 & 0 & 0 & 0 & 0 & 0 \\
0 & 0 & -1 & 1 & 0 & 0 & 0 & 0 \\
0 & 0 & 0 & 0 & 0 & 1 & -1 & 0 \\
0 & 0 & 0 & 0 & 0 & 0 & -1 & 1
\end{array}\right) .
$$

In the following examples (a) and (b), we focus on the type (I) flight states in Table 3 to avoid a crash (all flight states in Table 2 are achieved) when some motors fail and stop. These examples prove that Method 1 achieves the multirotor flight states to avoid crashes (type (I) in Table 3) using the remaining motors of the multirotor in the case of complete propeller motor failures.

(a) Under the conditions for the hexarotor, i.e., $t(s) \in(05), \theta=\phi=0(\mathrm{rad}), \ddot{\theta}=\ddot{\phi}=0\left(\mathrm{rad} / \mathrm{s}^{2}\right)$, $k_{F 1}=k_{F 2}=k_{F 4}=k_{F 5}=k_{F 6}=1.79 \times 10^{-7}\left(\mathrm{~N} / \mathrm{rpm}^{2}\right), k_{M 1}=k_{M 2}=k_{M 4}=k_{M 5}=k_{M 6}=4.38 \times 10^{-9}$ $\left(\mathrm{Nm} / \mathrm{rpm}^{2}\right)$, and $c$ (or $\left.\ddot{r}_{3}\right)=0\left(\mathrm{~m} / \mathrm{s}^{2}\right)$, when the motor of the third rotor has completely failed (Fig. 9), we ascertained the simulation result of the flight state to avoid a crash (type (I) in Table 3 and maneuver (ii) in Table 2) achieved by the motor speed control signals of $\omega_{M 1}=\omega_{M 2}=\omega_{M 4}$ $=\omega_{M 5}=5233.8205(\mathrm{rpm})$ and $\omega_{M 6}=0(\mathrm{rpm})$ from Eqs. (48)-(51), as shown in Figs. 10 and 11. $\psi(t), \theta(t)$, and $\phi(t)$ in Fig. 10 completely overlap. $r_{1}(t)$ and $r_{2}(t)$ in Fig. 11 also completely overlap.

In this case, the function $\boldsymbol{F}\left(\boldsymbol{x}, \boldsymbol{u}_{2 p-n}^{i_{1}, i_{2}, \ldots, i_{n}}\right)$ in Method 1 is as follows:

$$
\begin{gathered}
\boldsymbol{F}\left(\boldsymbol{\eta}, \boldsymbol{u}_{5}^{3}\right)=\boldsymbol{A}_{5}^{3}(\boldsymbol{\eta}) \boldsymbol{u}_{5}^{3}-\boldsymbol{b}_{5}, \\
\boldsymbol{A}_{5}^{3}(\boldsymbol{\eta}) \in \mathbb{R}^{5 \times 5}=\left(\begin{array}{c}
\boldsymbol{A}^{\prime}{ }_{4 \times 5}(\boldsymbol{\eta}) \\
\boldsymbol{\varepsilon}_{Q 2}^{\mathrm{T}} \boldsymbol{Q}_{2 \times 5}^{3}
\end{array}\right),
\end{gathered}
$$




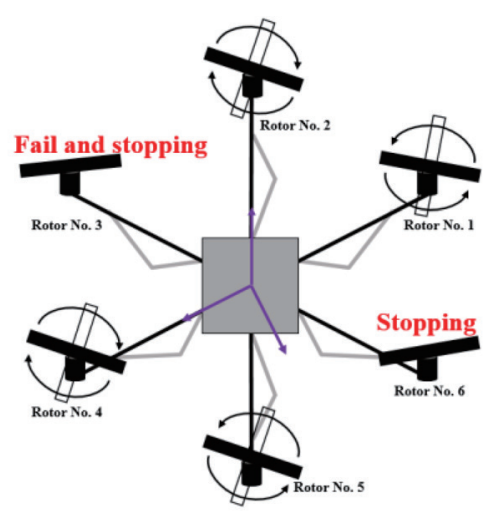

Fig. 9. (Color online) Example (a) of a hexarotor flight when the motor of the third rotor has completely failed.

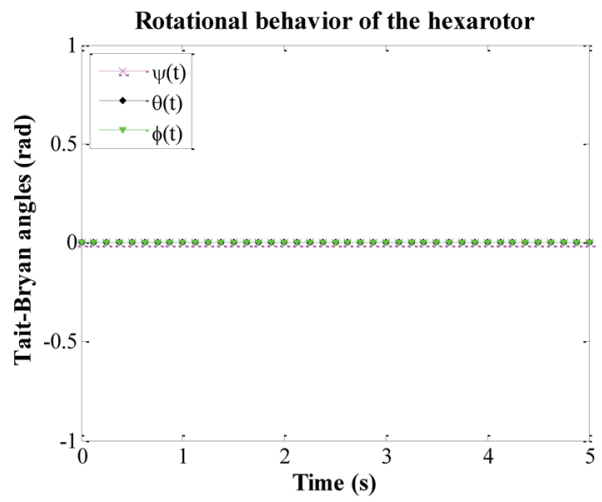

Fig. 10. (Color online) Simulation results of TaitBryan angles when the motor of the third rotor has completely failed [example (a)].

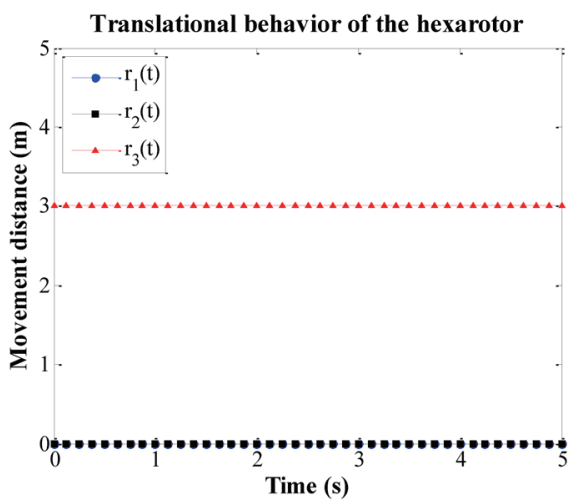

Fig. 11. (Color online) Simulation results of positions of motion when the motor of the third rotor has completely failed $\left(r_{3}=3(\mathrm{~m})\right.$ : initial condition) [example (a)].

$$
\begin{gathered}
\boldsymbol{A}^{\prime}{ }_{4 \times 5}(\boldsymbol{\eta}) \in \mathbb{R}^{4 \times 5}=\left(\begin{array}{c}
\boldsymbol{Z}(\boldsymbol{\eta}) \boldsymbol{S}_{\text {rot5 }}^{3} \\
\boldsymbol{e}_{3}^{\mathrm{T}} \frac{1}{m} \boldsymbol{B}(\boldsymbol{x}) \boldsymbol{S}_{\text {tra5 }}^{3}
\end{array}\right), \\
\boldsymbol{b}_{5}=\left(\ddot{\psi}, \ddot{\theta}, \ddot{\phi}, c+g, b_{1}^{\prime}\right)^{\mathrm{T}} \in \mathbb{R}^{5},
\end{gathered}
$$

where $i_{1}=3, n=1$, and $\boldsymbol{u}_{5}^{3} \in \mathbb{R}^{5} . \operatorname{span}\left\{\boldsymbol{\varepsilon}_{Q 1}, \boldsymbol{\varepsilon}_{Q 2}\right\}$ are the basis vectors of a right-handed twodimensional real vector space $\mathbb{R}^{2} . \boldsymbol{Q}_{2 \times 5}^{3} \in \mathbb{R}^{2 \times 5}$ is the constant matrix $\boldsymbol{Q}_{2 \times 6}$ with the 3rd row deleted. $b_{1}^{\prime} \in \mathbb{R}$ is a constant. $\boldsymbol{S}_{\xi 5}^{3}$ is the matrix $\boldsymbol{S}_{\xi 6}$ with the 3 rd row deleted ( $\xi=$ rot, tra).

(b) Under the conditions for the octorotor, i.e., $t(s) \in(05), \theta=0.08727$ (rad), $\phi=0$ (rad), $\ddot{\theta}=\ddot{\phi}=0\left(\mathrm{rad} / \mathrm{s}^{2}\right), k_{F 1}=k_{F 3}=k_{F 4}=k_{F 6}=k_{F 7}=1.79 \times 10^{-7}\left(\mathrm{~N} / \mathrm{rpm}^{2}\right), k_{M 1}=k_{M 3}=k_{M 4}=k_{M 6}=$ $k_{M 7}=4.38 \times 10^{-9}\left(\mathrm{Nm} / \mathrm{rpm}^{2}\right)$, and $c\left(\right.$ or $\left.\ddot{r}_{3}\right)=0\left(\mathrm{~m} / \mathrm{s}^{2}\right)$, when the motors of the second, fifth, and 
eighth rotors have completely failed (Fig. 12), we ascertained the simulation result of the flight state to avoid a crash (type (I) in Table 3, maneuver (iv-1) in Table 2, and $\theta$ is fixed) achieved by the motor speed control signals of $\omega_{M 1}=\omega_{M 6}=8249.4166(\mathrm{rpm}), \omega_{M 3}=\omega_{M 4}=5309.2771(\mathrm{rpm})$, and $\omega_{M 7}=0(\mathrm{rpm})$ from Eqs. (52)-(55), as shown in Figs. 13 and 14. $\psi(t)$ and $\phi(t)$ in Fig. 13 completely overlap.

In this case, the function $\boldsymbol{F}\left(\boldsymbol{x}, \boldsymbol{u}_{2 p-n}^{i_{1}, i_{2}, \ldots, i_{n}}\right)$ in Method 1 is as follows:

$$
\begin{gathered}
\boldsymbol{F}\left(\boldsymbol{\eta}, \boldsymbol{u}_{5}^{2,5,8}\right)=\boldsymbol{A}_{5}^{2,5,8}(\boldsymbol{\eta}) \boldsymbol{u}_{5}^{2,5,8}-\boldsymbol{b}_{5}, \\
\boldsymbol{A}_{5}^{2,5,8}(\boldsymbol{\eta}) \in \mathbb{R}^{5 \times 5}=\left(\begin{array}{c}
\boldsymbol{A}^{\prime 2,5,8}(\boldsymbol{\eta}) \\
\boldsymbol{\varepsilon}_{Q 2}^{\mathrm{T}} \boldsymbol{Q}_{4 \times 5}^{2,5,8}
\end{array}\right), \\
\boldsymbol{A}_{4 \times 5}^{\prime 2,5,8}(\boldsymbol{\eta}) \in \mathbb{R}^{4 \times 5}=\left(\begin{array}{c}
\boldsymbol{Z}(\boldsymbol{\eta}) \boldsymbol{S}_{\mathrm{rot}}^{2,5,8} \\
\boldsymbol{e}_{3}^{\mathrm{T}} \frac{1}{m} \boldsymbol{B}(\boldsymbol{x}) \boldsymbol{S}_{\mathrm{tra} 5}^{2,5,8}
\end{array}\right),
\end{gathered}
$$

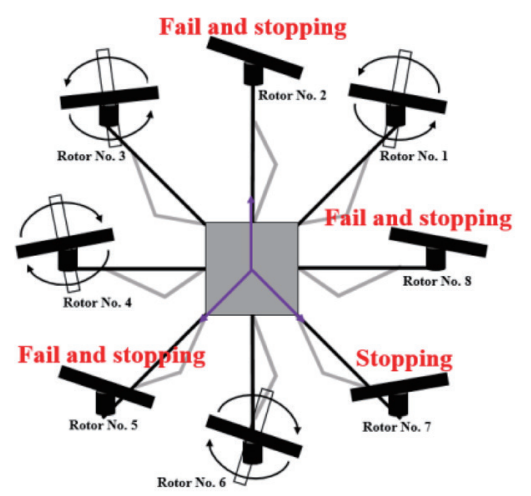

Fig. 12. (Color online) Example (b) of an octorotor flight when the motors in the second, fifth, and eighth rotors have completely failed.

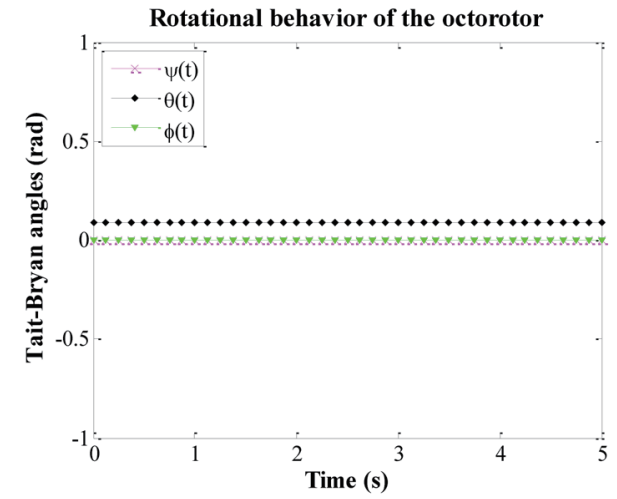

Fig. 13. (Color online) Simulation results of TaitBryan angles when the motors of the second, fifth, and eighth rotors have completely failed [example (b)].

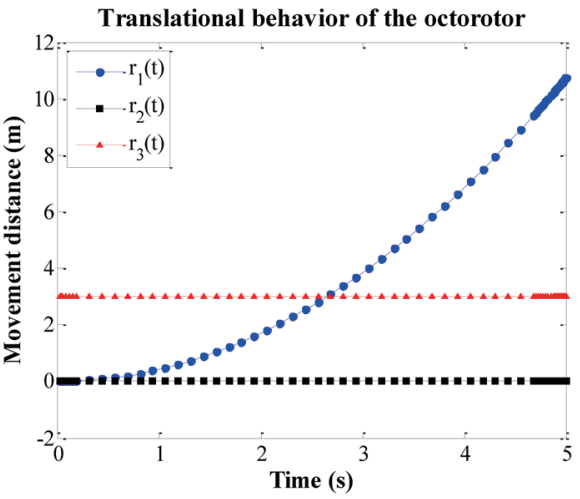

Fig. 14. (Color online) Simulation results of positions of motion when the motors of the second, fifth, and eighth rotors have completely failed $\left(r_{3}=3(\mathrm{~m})\right.$ : initial condition) [example (b)]. 


$$
\boldsymbol{b}_{5}=\left(\ddot{\psi}, \ddot{\theta}, \ddot{\phi}, c+g, b_{1}^{\prime}\right)^{\mathrm{T}} \in \mathbb{R}^{5},
$$

where $i_{1}=2, i_{2}=5, i_{3}=8$, and $n=3 . \boldsymbol{u}_{5}^{2,5,8} \in \mathbb{R}^{5} . \operatorname{span}\left\{\boldsymbol{\varepsilon}_{Q 1}, \boldsymbol{\varepsilon}_{Q 2}, \boldsymbol{\varepsilon}_{Q 3}, \boldsymbol{\varepsilon}_{Q 4}\right\}$ are the basis vectors of a right-handed four-dimensional real vector space $\mathbb{R}^{4} \cdot \boldsymbol{Q}_{4 \times 5}^{2,5,8} \in \mathbb{R}^{4 \times 5}$ is the constant matrix $\boldsymbol{Q}_{4 \times 8}$ with the 2nd, 5th, and 8th rows deleted. $b_{1}^{\prime} \in \mathbb{R}$ is a constant. $\boldsymbol{S}_{\xi 5}^{2,5,8}$ is the matrix $\boldsymbol{S}_{\xi 8}$ with the 2 nd, 5 th, and 8 th rows deleted ( $\xi=$ rot, tra).

In the following examples (c) to (e), we also focus on the type (II) flight in Table 3 to avoid a crash (yaw angles are freely turning) when some motors fail and stop. Although rotational accelerations of the yaw angle are observed, examples (c) to (e) prove that Method 1 also achieves the multirotor flight states to avoid crashes (type (II) in Table 3) using the remaining motors of the multirotor in the case of complete propeller motor failures.

(c) Under the conditions for the quadrotor, i.e., $t(s) \in(05), \theta=\phi=0(\mathrm{rad}), \ddot{\theta}=\ddot{\phi}=0$ $\left(\mathrm{rad} / \mathrm{s}^{2}\right), k_{F 1}=k_{F 3}=k_{F 4}=1.79 \times 10^{-7}\left(\mathrm{~N} / \mathrm{rpm}^{2}\right), k_{M 1}=k_{M 3}=k_{M 4}=4.38 \times 10^{-9}\left(\mathrm{Nm} / \mathrm{rpm}^{2}\right)$, and $c\left(\right.$ or $\left.\ddot{r}_{3}\right)=0\left(\mathrm{~m} / \mathrm{s}^{2}\right)$, when the motor of the second rotor has completely failed (Fig. 15), we ascertained the simulation result of the flight state to avoid a crash (type (II) in Table 3, maneuvers (i-1) and (i-2) in Table $2, \ddot{r}_{3}$ is fixed, and $\ddot{\psi}=-12.3371\left(\mathrm{rad} / \mathrm{s}^{2}\right)$ as a result) achieved by the motor speed control signals of $\omega_{M 1}=\omega_{M 3}=6735.1766(\mathrm{rpm})$ and $\omega_{M 4}=0(\mathrm{rpm})$ from Eqs. (56)-(58), as shown in Figs. 16 and 17. $\theta(t)$ and $\phi(t)$ in Fig. 16 completely overlap. $r_{1}(t)$ and $r_{2}(t)$ in Fig. 17 also completely overlap.

In this case, the function $\boldsymbol{F}\left(\boldsymbol{x}, \boldsymbol{u}_{2 p-n}^{i_{1}, i_{2}, \ldots, i_{n}}\right)$ in Method 1 is as follows:

$$
\begin{gathered}
\boldsymbol{F}\left(\boldsymbol{\eta}, \boldsymbol{u}_{3}^{2}\right)=\boldsymbol{A}_{3}^{2}(\boldsymbol{\eta}) \boldsymbol{u}_{3}^{2}-\boldsymbol{b}_{3}, \\
\boldsymbol{A}_{3}^{2}(\boldsymbol{\eta}) \in \mathbb{R}^{3 \times 3}=\left(\begin{array}{c}
\boldsymbol{\varepsilon}_{2}^{\mathrm{T}} \boldsymbol{Z}(\boldsymbol{\eta}) \boldsymbol{S}_{\mathrm{rot} 3}^{2} \\
\boldsymbol{\varepsilon}_{3}^{\mathrm{T}} \boldsymbol{Z}(\boldsymbol{\eta}) \boldsymbol{S}_{\mathrm{rot} 3}^{2} \\
\boldsymbol{e}_{3}^{\mathrm{T}} \frac{1}{m} \boldsymbol{B}(\boldsymbol{x}) \boldsymbol{S}_{\mathrm{tra} 3}^{2}
\end{array}\right),
\end{gathered}
$$

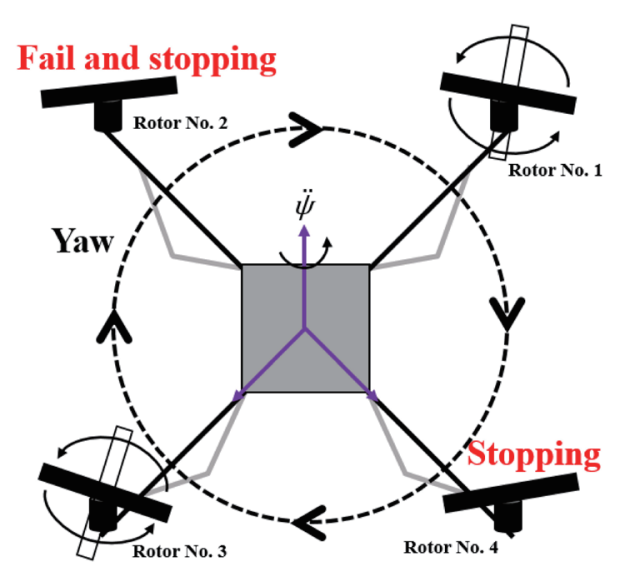

Fig. 15. (Color online) Example (c) of a quadrotor flight when the motor of the second rotor has completely failed. 


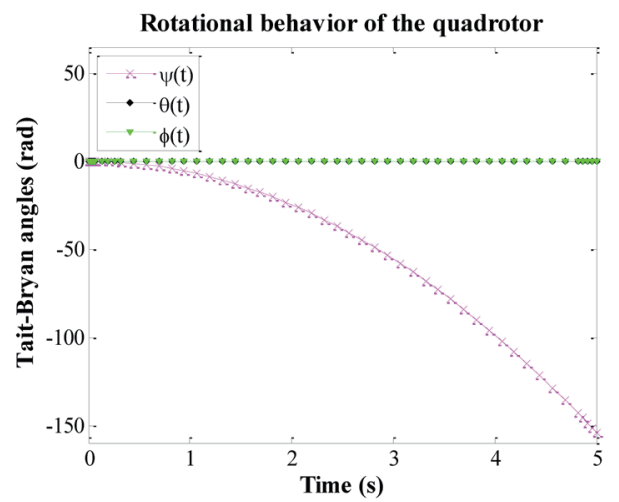

Fig. 16. (Color online) Simulation results of TaitBryan angles when the motor of the second rotor has completely failed [example (c)].

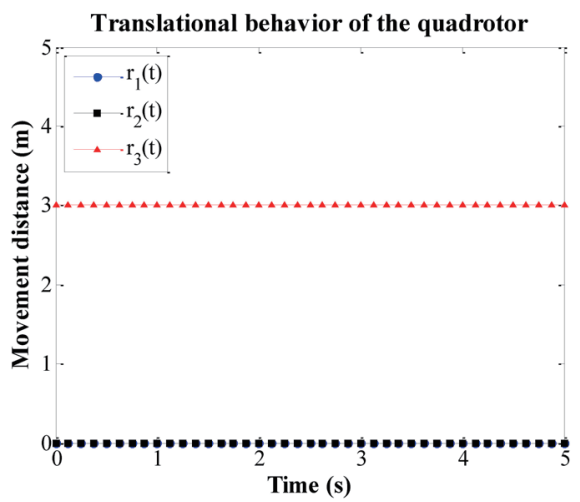

Fig. 17. (Color online) Simulation results of positions of motion when the motor of the second rotors has completely failed $\left(r_{3}=3(\mathrm{~m})\right.$ : initial condition) [example (c)].

$$
\boldsymbol{b}_{3}=(\ddot{\theta}, \ddot{\phi}, c+g)^{\mathrm{T}} \in \mathbb{R}^{3},
$$

where $i_{1}=2, n=1$, and $\boldsymbol{u}_{3}^{2} \in \mathbb{R}^{3} . \boldsymbol{S}_{\xi 3}^{2}$ is the matrix $\boldsymbol{S}_{\xi 4}$ with the 2 nd row deleted ( $\xi=$ rot, tra).

(d) Under the conditions for the quadrotor, i.e., $t(s) \in(00.1), \theta=\phi=0(\mathrm{rad}), \ddot{\theta}=0\left(\mathrm{rad} / \mathrm{s}^{2}\right)$, $\ddot{\phi}=17.4533\left(\mathrm{rad} / \mathrm{s}^{2}\right), k_{F 2}=k_{F 4}=1.79 \times 10^{-7}\left(\mathrm{~N} / \mathrm{rpm}^{2}\right), k_{M 2}=k_{M 4}=4.38 \times 10^{-9}\left(\mathrm{Nm} / \mathrm{rpm}^{2}\right)$, and $c\left(\right.$ or $\left.\ddot{r}_{3}\right)=0\left(\mathrm{~m} / \mathrm{s}^{2}\right)$, when the motors of the first and third rotors have completely failed (Fig. 18), we ascertained the simulation result of the flight state to avoid a crash (type (II) in Table 3, maneuver (v-2) in Table 2, and $\ddot{\psi}=12.3371\left(\mathrm{rad} / \mathrm{s}^{2}\right)$ as a result) achieved by the motor speed control signals of $\omega_{M 2}=6535.6936(\mathrm{rpm})$ and $\omega_{M 4}=6928.9189$ (rpm) from Eqs. (59)-(61), as shown in Figs. 19 and 20. $r_{1}(t)$ and $r_{2}(t)$ in Fig. 20 completely overlap. Note that translational

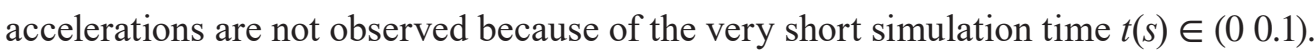

In this case, the function $\boldsymbol{F}\left(\boldsymbol{x}, \boldsymbol{u}_{2 p-n}^{i_{1}, i_{2}, \ldots, i_{n}}\right)$ in Method 1 is as follows:

$$
\begin{gathered}
\boldsymbol{F}\left(\boldsymbol{\eta}, \boldsymbol{u}_{2}^{1,3}\right)=\boldsymbol{A}_{2}^{1,3}(\boldsymbol{\eta}) \boldsymbol{u}_{2}^{1,3}-\boldsymbol{b}_{2}, \\
\boldsymbol{A}_{2}^{1,3}(\boldsymbol{\eta}) \in \mathbb{R}^{2 \times 2}=\left(\begin{array}{c}
\boldsymbol{\varepsilon}_{3}^{\mathrm{T}} \boldsymbol{Z}(\boldsymbol{\eta}) \boldsymbol{S}_{\mathrm{rot} 2}^{1,3} \\
\boldsymbol{e}_{3}^{\mathrm{T}} \frac{1}{m} \boldsymbol{B}(\boldsymbol{x}) \boldsymbol{S}_{\text {tra } 2}^{1,3}
\end{array}\right), \\
\boldsymbol{b}_{2}=(\ddot{\phi}, c+g)^{\mathrm{T}} \in \mathbb{R}^{2} .
\end{gathered}
$$

where $i_{1}=1, i_{2}=3, n=2$, and $\boldsymbol{u}_{2}^{1,3} \in \mathbb{R}^{2} . \boldsymbol{S}_{\xi 2}^{1,3}$ is the matrix $\boldsymbol{S}_{\xi 4}$ with the 1 st and 3rd rows deleted $(\xi=$ rot, tra).

(e) Under the conditions for the octorotor, i.e., $t(s) \in(0$ 5), $\theta=\phi=0$ (rad), $\ddot{\theta}=\ddot{\phi}=0$ $\left(\mathrm{rad} / \mathrm{s}^{2}\right), k_{F 2}=k_{F 5}=k_{F 7}=1.79 \times 10^{-7}\left(\mathrm{~N} / \mathrm{rpm}^{2}\right), k_{M 2}=k_{M 5}=k_{M 7}=4.38 \times 10^{-9}\left(\mathrm{Nm} / \mathrm{rpm}^{2}\right)$, and $c$ (or $\left.\ddot{r}_{3}\right)=-0.1\left(\mathrm{~m} / \mathrm{s}^{2}\right)$, when the motors of the first, third, fourth, sixth, and eighth rotors 


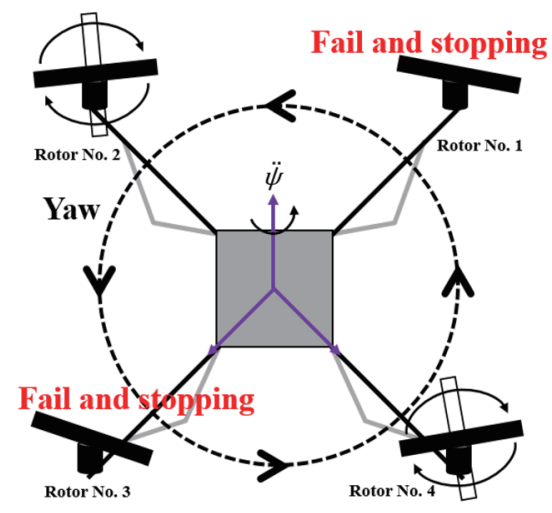

Fig. 18. (Color online) Example (d) of a quadrotor flight when the motors of the first and third rotors have completely failed.

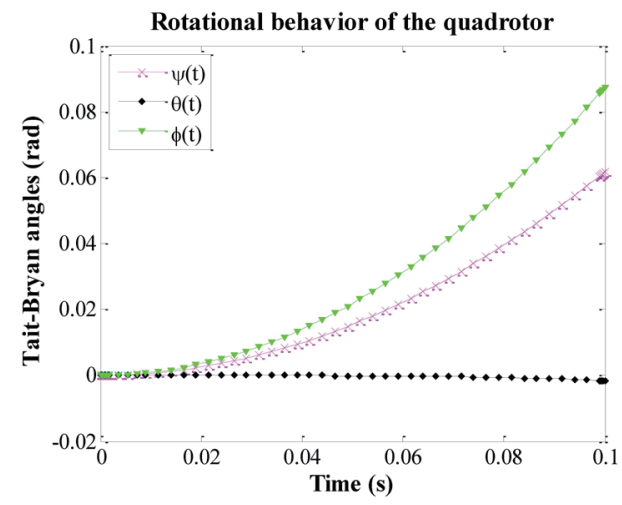

Fig. 19. (Color online) Simulation results of TaitBryan angles when the motors of the first and third rotors have completely failed (example (d)).

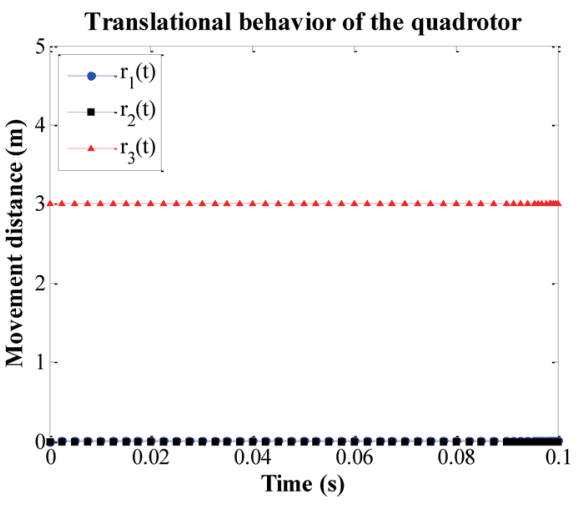

Fig. 20. (Color online) Simulation results of positions of motion when the motors of the first and third rotors have completely failed $\left(r_{3}=3(\mathrm{~m})\right.$ : initial condition) (example (d)).

have completely failed (Fig. 21), we ascertained the simulation result of the flight state to avoid a crash (type (II) in Table 3, maneuver (i-2) in Table 2, and $\ddot{\psi}=-2.2140\left(\mathrm{rad} / \mathrm{s}^{2}\right.$ ) as a result) achieved by the motor speed control signals of $\omega_{M 2}=8866.5435(\mathrm{rpm})$ and $\omega_{M 5}=\omega_{M 7}=7455.8446(\mathrm{rpm})$ from Eqs. (62)-(64), as shown in Figs. 22 and 23. $\theta(t)$ and $\phi(t)$ in Fig. 22 completely overlap. $r_{1}(t)$ and $r_{2}(t)$ in Fig. 23 also completely overlap.

In this case, the function $\boldsymbol{F}\left(\boldsymbol{x}, \boldsymbol{u}_{2 p-n}^{i_{1}, i_{2}, \ldots, i_{n}}\right)$ in Method 1 is as follows:

$$
\begin{gathered}
\boldsymbol{F}\left(\boldsymbol{\eta}, \boldsymbol{u}_{3}^{1,3,4,6,8}\right)=\boldsymbol{A}_{3}^{1,3,4,6,8}(\boldsymbol{\eta}) \boldsymbol{u}_{3}^{1,3,4,6,8}-\boldsymbol{b}_{3}, \\
\boldsymbol{A}_{3}^{1,3,4,6,8}(\boldsymbol{\eta}) \in \mathbb{R}^{3 \times 3}=\left(\begin{array}{c}
\boldsymbol{\varepsilon}_{2}^{\mathrm{T}} \boldsymbol{Z}(\boldsymbol{\eta}) \boldsymbol{S}_{\text {rot3 }}^{1,3,4,6,8} \\
\boldsymbol{\varepsilon}_{3}^{\mathrm{T}} \boldsymbol{Z}(\boldsymbol{\eta}) \boldsymbol{S}_{\text {rot }}^{1,3,4,4,8} \\
\boldsymbol{e}_{3}^{\mathrm{T}} \frac{1}{m} \boldsymbol{B}(\boldsymbol{x}) \boldsymbol{S}_{\text {tra3 }}^{1,3,4,6,8}
\end{array}\right),
\end{gathered}
$$




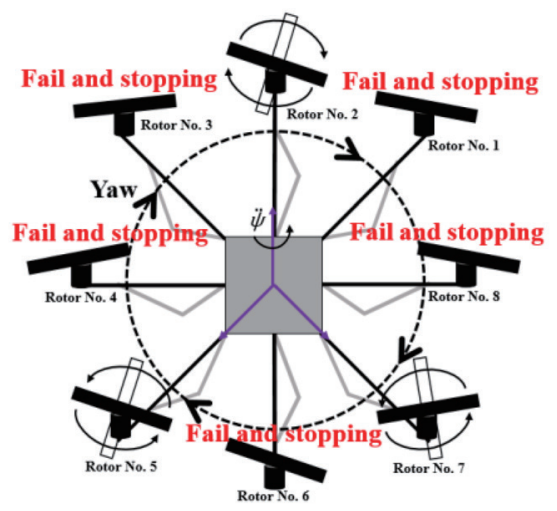

Fig. 21. (Color online) Example (e) of an octorotor flight when the motors of the first, third, fourth, sixth, and eighth rotors have completely failed.

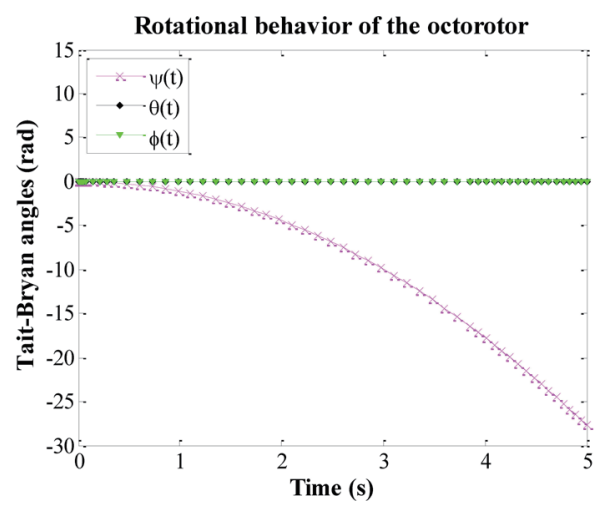

Fig. 22. (Color online) Simulation results of TaitBryan angles when the motors of the first, third, fourth, sixth, and eighth rotors have completely failed [example (e)].

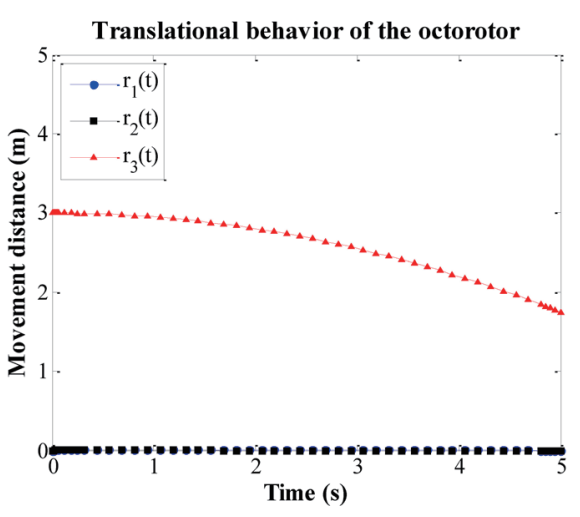

Fig. 23. (Color online) Simulation results of positions of motion when the motors of the first, third, fourth, sixth, and eighth rotors have completely failed $\left(r_{3}=3(\mathrm{~m})\right.$ : initial condition) [example (e)].

$$
\boldsymbol{b}_{3}=(\ddot{\theta}, \ddot{\phi}, c+g)^{\mathrm{T}} \in \mathbb{R}^{3},
$$

where $i_{1}=1, i_{2}=3, i_{3}=4, i_{4}=6, i_{5}=8, n=5$, and $\boldsymbol{u}_{3}^{1,3,4,6,8} \in \mathbb{R}^{3} . \boldsymbol{S}_{\xi 3}^{1,3,4,6,8}$ is the matrix $\boldsymbol{S}_{\xi 8}$ with the 1st, 3rd, 4th, 6th, and 8th rows deleted ( $\xi=$ rot, tra).

\section{Conclusions}

The following results were obtained:

1) By using the state variables of the Euler angle rotational motion $\boldsymbol{x}=(\psi, \theta, \phi)^{\mathrm{T}}$, $\dot{\boldsymbol{x}}=(\dot{\psi}, \dot{\theta}, \dot{\phi})^{\mathrm{T}}$ and translational motion $\boldsymbol{r}=\left(r_{1}, r_{2}, r_{3}\right)^{\mathrm{T}}, \dot{\boldsymbol{r}}=\left(\dot{r}_{1}, \dot{r}_{2}, \dot{r}_{3}\right)^{\mathrm{T}}$ (Fig. 5), we have summarized the multirotor maneuvers and flight states in many applications in Table 2 (Sect. 2.3) and two types of multirotor flight states to avoid a crash when some motors fail and stop in Table 3 (Sect. 3). 
2) We have related the operating and equilibrium points of multirotors to the flight states in Table 2 and provided definitions of the operating and equilibrium points of multirotors.

3) We have provided definitions of multirotor motor speed control signal vectors and Method 1 (Sect. 3) of directly providing motor speed control signals for multirotor flights in the case of complete motor failures derived from Theorem 4 (Appendix 3), such that the type (I) multirotor flight state in Table 3 to avoid a crash (Sect. 3) is achieved, and Theorem 5 (in Appendix 3), such that the type (II) multirotor flight state in Table 3 to avoid a crash is achieved.

4) In Sect. 4, we have illustrated typical examples of the two types of multirotor flight states in Table 3 to avoid a crash obtained by Method 1. Method 1 with examples of the two types of multirotor flight states to avoid a crash, as shown in Table 3, could lead to design guidelines regarding what kind of configuration, such as the choice or setting of the motor, propeller, or electronic speed controller sets, is most stable for the flight states to avoid a crash when some motors fail and stop.

5) In Appendix 1, we have provided dynamical Euler angle state equations of rotations for a multirotor as Theorem 1 and dynamical state equations depending on Euler angles of translations for the multirotor as Theorem 2. It is noteworthy that the function on the righthand side of Eq. (84) in Theorem 1 has no $\psi$ state variable.

6) In Appendix 2, we have provided maneuverable flight control methods of Theorem 3, including how to control motors to achieve the multirotor maneuvers and flight states of Fig. 5 in Table 2.

7) We will verify Method 1 experimentally through several tests of actual multirotor flights to avoid a crash in the case of complete propeller failures using a commercial multirotor model with modifications based on Method 1 employing the examples in Sect. 4 as a guideline.

8) Using Method 1 with the examples in Sect. 4 as a guideline, we will build a state variable feedback control for stabilizing the flight states (or flight operating points) of a multirotor under the influence of disturbances such as wind.

\section{Acknowledgments}

We give our special thanks to the mathematician Dr. Keisuke Kikuchi of Shonan Institute of Technology for his many helpful insights.

\section{References}

1 R. Mahony, V. Kumar, and P. Corke: IEEE Robot Autom. Mag. 19 (2012) 20. https://doi.org/10.1109/ MRA.2012.2206474

2 T. Du, A. Schulz, B. Zhu, B. Bickel, and W. Matusik: ACM Trans. Graphics 35 (2016) 227. https://doi. org/10.1145/2980179.2982427

3 P. Castillo, R. Lozano, and A. Dzul: IEEE Control Syst. Mag. 25 (2005) 45. https://doi.org/10.1109/ MCS.2005.1550152

4 D. Mellinger, N. Michael, and V. Kumar: Int. J. Rob. Res. 31 (2012) 664. https://doi. org $/ 10.1177 / 0278364911434236$

5 H. Okazaki, K. Isogai, and H. Nakano: Proc. 2016 IEEE 59th Int. Midwest Symp. Circuits and Systems (IEEE, 2016) 117. https://doi.org/10.1109/MWSCAS.2016.7869969 
6 K. Isogai, H. Nakano, and H. Okazaki: Proc. 2018 IEEE 61st Int. Midwest Symp. Circuits and Systems (IEEE, 2018) 388. https://doi.org/10.1109/MWSCAS.2018.8623851

7 H. Okazaki, S. Yin, K. Isogai, and H. Nakano: Proc. 2018 IEEE 61st Int. Midwest Symp. Circuits and Systems (IEEE, 2018) 384. https://doi.org/10.1109/MWSCAS.2018.8623843

8 A. Freddi, A. Lanzon, and S. Longhi: Proc. 18th IFAC World Congress (IFAC, 2011) 5413. https://doi. org/10.3182/20110828-6-IT-1002.02016

9 M. W. Mueller and R. D'Andrea: Proc. 2014 IEEE Int. Conf. Robotics \& Automation (IEEE, 2014) 45. https:// doi.org/10.1109/ICRA.2014.6906588

10 S. Dongjie, Y. Binxian, and Q. Quan: Proc. 35th Chinese Control Conf. (IEEE, 2016) 6740. https://doi. org/10.1109/ChiCC.2016.7554418

11 M. Saied, B. Lussier, I. Fantoni, H. Shraim, and C. Francis: Proc. 20th IFAC World Congress (IFAC, 2017) 5263.https://doi.org/10.1016/j.ifacol.2017.08.468

12 F. C. Moon: Applied Dynamics: With Applications to Multibody and Mechatronic Systems (John Wiley and Sons Inc., New York, 1998) pp. 103-167, 168-253, and 474-477.

13 H. Goldstein: Classical Mechanics (Addison-Wesley, Boston, 1980) 2nd ed., Chaps. 4 and 5.

14 V. I. Arnold: Mathematical Methods of Classical Mechanics (Springer-Verlag, New York, 1997) 2nd ed., Chap. 6.

15 Y. Yamamoto: From Vector Spaces to Function Spaces - Introduction to Functional Analysis with Applications - (SIAM, Philadelphia, 2012) pp. 203-207. https://doi.org/10.1137/9781611972313

16 D. J. Higham and N. J. Higham: MATLAB Guide (SIAM, Philadelphia, 2005) 2nd ed., pp. 175-184.

17 H. Okazaki, K. Yashikida, and H. Mizutani: Proc. 2012 IEEE 55th Int. Midwest Symp. Circuits and Systems (IEEE, 2012) 490. https://doi.org/10.1109/MWSCAS.2012.6292064

18 R. E. Kalman: SIAM J. Ser. A Control 1 (1963) 152. https://doi.org/10.1137/0301010

19 S. Kodama and N. Suda: Matrix Theory for Systems and Control (The Society of Instrument and Control Engineers of Japan, Tokyo, 1978) p. 248 (in Japanese).

20 E. Eriksson, D. Estep, and C. Johnson: Applied Mathematics: Body and Soul (Springer-Verlag, New York, 2004) vol. 2, pp. 621-626.

\section{About the Authors}

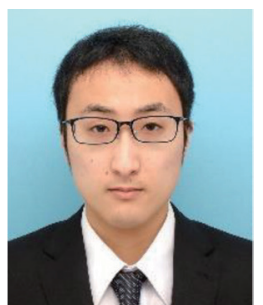

Kaito Isogai received his B.E. in applied computer science and his M.E. in electrical and information engineering from Shonan Institute of Technology (SIT) in 2015 and 2017, respectively. He is currently pursuing his Ph.D. degree at the Graduate School of Engineering, Electrical and Information Engineering of SIT. His research interests include nonlinear control, mechatronics, and robotics. (kaito.isogai.3@gmail.com)

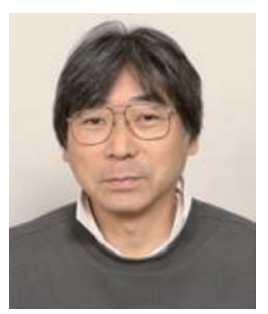

Hideo Nakano received his B.E. and M.E. degrees in mechanical engineering from Waseda University in 1977 and 1980, respectively. He was a research associate at Waseda University from 1983 to 1986 . He was a lecturer in the Department of Mechanical Engineering, Shonan Institute of Technology (SIT), from 1987 to 2001 and an associate professor in SIT from 2001 to 2005. From 2005 to 2014, he was an associate professor in the Department of Applied Computer Sciences at SIT and has been a professor from 2014 to the present, including the elected Department Head from 2015 to 2019. His research interests include modeling methodology and the dynamical behavior of complex systems. (nakano@sc.shonan-it.ac.jp) 


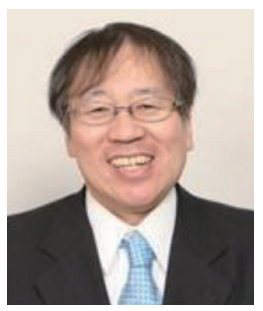

Hideaki Okazaki received his B.E., M.E., and Ph.D. degrees in mechanical engineering from Waseda University in 1984, 1986, and 1996, respectively. He was a research associate at Waseda University from 1989 to 1992, an assistant professor of the Department of Electronic Control Engineering, Gifu National College of Technology, from 1992 to 1996, and an associate professor of the Department of Electronic Control Engineering, Gifu National College of Technology, from 1996 to 2000 . He was engaged in applied mathematics as a visiting scientist at Cornell University, USA from 1999 to 2001 . He was made a professor in the Department of System and Communication Engineering, Shonan Institute of Technology (SIT), from 2001 to 2005. From 2006 to the present, he has been a professor in the Department of Applied Computer Sciences at SIT, and from 2013 to the present, he has been a professor in the Graduate School of Engineering, Electrical and Information Engineering at SIT. He has recently been made Director of Internet of Things-Inspired Interdisciplinary Research Center (IoT-IIRC) at SIT. His research interests include nonlinear system analysis and control and methods of rigorous computer-assisted proof for nonlinear systems.

(okazaki@sc.shonan-it.ac.jp)

\section{Appendix 1: Dynamical System State Equations of a Multirotor}

In this appendix, we provide a theorem of the dynamical Euler angle state equations of rotations for a multirotor and a theorem of the dynamical state equations of translations for a multirotor.

\subsection{Euler-Lagrange equations for a multirotor with Euler angle state variables}

\subsubsection{Euler angle variables and multirotor Lagrangian}

By using the basis vectors of a right-handed three-dimensional real vector space $\mathbb{R}^{3}$ (i.e., orthogonal space) $\operatorname{span}\left\{\boldsymbol{\varepsilon}_{1}, \boldsymbol{\varepsilon}_{2}, \boldsymbol{\varepsilon}_{3}\right\}, \boldsymbol{x}=(\psi, \theta, \phi)^{\mathrm{T}}$ and $\dot{\boldsymbol{x}}=(\dot{\psi}, \dot{\theta}, \dot{\phi})^{\mathrm{T}}$ are described by Eqs. (65) and (66), respectively.

$$
\begin{gathered}
\boldsymbol{x}=\psi \boldsymbol{\varepsilon}_{1}+\theta \boldsymbol{\varepsilon}_{2}+\phi \boldsymbol{\varepsilon}_{3}=\psi \boldsymbol{\varepsilon}_{1}+\boldsymbol{\eta}, \\
\dot{\boldsymbol{x}}=\dot{\psi} \boldsymbol{\varepsilon}_{1}+\dot{\theta} \boldsymbol{\varepsilon}_{2}+\dot{\phi} \boldsymbol{\varepsilon}_{3}=\dot{\psi} \boldsymbol{\varepsilon}_{1}+\dot{\boldsymbol{\eta}}, \\
\boldsymbol{\eta}=\theta \boldsymbol{\varepsilon}_{2}+\phi \boldsymbol{\varepsilon}_{3}, \\
\dot{\boldsymbol{\eta}}=\dot{\theta} \boldsymbol{\varepsilon}_{2}+\dot{\phi} \boldsymbol{\varepsilon}_{3} .
\end{gathered}
$$


Taking the instantaneous angular velocity $\Omega \in W$ and the translational variables $\boldsymbol{r}$ and $\dot{\boldsymbol{r}}$ as the state variables of a multirotor, we define the Lagrangian of the multirotor as

$$
L=\frac{1}{2} m\langle\dot{\boldsymbol{r}}, \dot{\boldsymbol{r}}\rangle+\frac{1}{2}\langle\hat{\boldsymbol{I}} \boldsymbol{\Omega}, \boldsymbol{\Omega}\rangle-m g\left\langle\boldsymbol{r}, \boldsymbol{e}_{3}\right\rangle,
$$

where $m$ is the total mass of the multirotor and $g$ is the gravitational acceleration.

\subsubsection{Equations of rotations}

In terms of generalized velocities, the virtual power in Ref. 12 of outside generalized forces (or so-called moments) $\boldsymbol{B}(\boldsymbol{x}) \boldsymbol{F}_{\text {rot }}(\boldsymbol{u}), \boldsymbol{F}_{\text {rot }}(\boldsymbol{u})=\boldsymbol{S}_{\text {rot } 2 p} \boldsymbol{u}, \boldsymbol{u} \in \Lambda$ is given as

$$
\begin{aligned}
\left\langle\boldsymbol{B}(\boldsymbol{x}) \boldsymbol{F}_{\mathrm{rot}}(\boldsymbol{u}), \delta \boldsymbol{\omega}\right\rangle & =\left\langle\boldsymbol{B}(\boldsymbol{x}) \boldsymbol{F}_{\mathrm{rot}}(\boldsymbol{u}), \omega_{\dot{\boldsymbol{x}}}(\psi, \theta) \delta \dot{\boldsymbol{x}}\right\rangle \\
& =\left\langle\omega_{\dot{\boldsymbol{x}}}(\psi, \theta)^{\mathrm{T}} \boldsymbol{B}(\boldsymbol{x}) \boldsymbol{F}_{\mathrm{rot}}(\boldsymbol{u}), \delta \dot{\boldsymbol{x}}\right\rangle,
\end{aligned}
$$

where $\boldsymbol{\omega}_{\dot{\boldsymbol{x}}}(\psi, \theta)^{\mathrm{T}} \boldsymbol{B}(\boldsymbol{x}) \boldsymbol{F}_{\text {rot }}(\boldsymbol{u})$ refers to the generalized forces and $\boldsymbol{u} \in \Lambda \subset \mathbb{R}^{\rho}$ with $\rho \in \mathbb{N}$ being the $\rho$-parameter vector of the inputs of the multirotor that control the outer generalized forces acting on the multirotor. ${ }^{(18)}$

Hence, taking $\boldsymbol{x}$ and $\dot{\boldsymbol{x}}$ as the state variables of the multirotor rotations, we obtain the EulerLagrange equation of rotation for the multirotor as follows:

$$
\begin{gathered}
L=\frac{1}{2} m\langle\dot{\boldsymbol{r}}, \dot{\boldsymbol{r}}\rangle+\frac{1}{2}\left\langle\hat{\boldsymbol{I}} \boldsymbol{B}(\boldsymbol{x})^{\mathrm{T}} \boldsymbol{\omega}(\psi, \theta, \dot{\boldsymbol{x}}), \boldsymbol{B}(\boldsymbol{x})^{\mathrm{T}} \boldsymbol{\omega}(\psi, \theta, \dot{\boldsymbol{x}})\right\rangle-m g\left\langle\boldsymbol{r}, \boldsymbol{e}_{3}\right\rangle \\
=\frac{1}{2} m\langle\dot{\boldsymbol{r}}, \dot{\boldsymbol{r}}\rangle+\frac{1}{2}\left\langle\hat{\boldsymbol{I}} \boldsymbol{B}(\boldsymbol{x})^{\mathrm{T}} \boldsymbol{\omega}_{\dot{\boldsymbol{x}}}(\psi, \theta) \cdot \dot{\boldsymbol{x}}, \boldsymbol{B}(\boldsymbol{x})^{\mathrm{T}} \omega_{\dot{\boldsymbol{x}}}(\psi, \theta) \cdot \dot{\boldsymbol{x}}\right\rangle-m g\left\langle\boldsymbol{r}, \boldsymbol{e}_{3}\right\rangle, \\
\frac{d}{d t} L_{\dot{\boldsymbol{x}}}(\boldsymbol{\eta}, \dot{\boldsymbol{x}}, \ddot{\boldsymbol{x}})-L_{\boldsymbol{x}}(\boldsymbol{\eta}, \dot{\boldsymbol{x}})=\boldsymbol{\omega}_{\dot{\boldsymbol{x}}}(\psi, \theta)^{\mathrm{T}} \boldsymbol{B}(\boldsymbol{x}) \boldsymbol{F}_{\mathrm{rot}}(\boldsymbol{u}), \\
\omega(\psi, \theta, \dot{\boldsymbol{x}})=\boldsymbol{B}(\boldsymbol{x}) \boldsymbol{\Omega}(\boldsymbol{\eta}, \dot{\boldsymbol{x}}) .
\end{gathered}
$$

Then, $\boldsymbol{\omega}(\psi, \theta, \dot{\boldsymbol{x}})$ and $\boldsymbol{\omega}_{\dot{\boldsymbol{x}}}(\psi, \theta)$ can be concretely expressed by

$$
\begin{gathered}
\omega(\psi, \theta, \dot{\boldsymbol{x}})=(-\dot{\theta} \sin \psi+\dot{\phi} \cos \theta \cos \psi) \boldsymbol{e}_{1}+(\dot{\theta} \cos \psi+\dot{\phi} \cos \theta \sin \psi) \boldsymbol{e}_{2}+(\dot{\psi}-\dot{\phi} \sin \theta) \boldsymbol{e}_{3}, \\
\omega_{\dot{x}}(\psi, \theta)=\left(\begin{array}{ccc}
0 & -\sin \psi & \cos \theta \cos \psi \\
0 & \cos \psi & \cos \theta \sin \psi \\
1 & 0 & -\sin \theta
\end{array}\right)
\end{gathered}
$$

In the following, the angles of $\boldsymbol{x}$ for operator $\boldsymbol{B}$ and the angles $\psi$ and $\theta$ for $\boldsymbol{\omega}_{\dot{\boldsymbol{x}}}$ are often omitted for convenience: 


$$
\begin{aligned}
& L_{\dot{x}_{k}}=\frac{1}{2}\left\langle\hat{\boldsymbol{I}} \boldsymbol{B}^{\mathrm{T}} \omega_{\dot{\boldsymbol{x}}} \boldsymbol{\varepsilon}_{k}, \boldsymbol{B}^{\mathrm{T}} \omega_{\dot{\boldsymbol{x}}} \dot{\boldsymbol{x}}\right\rangle+\frac{1}{2}\left\langle\hat{\boldsymbol{I}} \boldsymbol{B}^{\mathrm{T}} \boldsymbol{\omega}_{\dot{\boldsymbol{x}}} \dot{\boldsymbol{x}}, \boldsymbol{B}^{\mathrm{T}} \boldsymbol{\omega}_{\dot{\boldsymbol{x}}} \boldsymbol{\varepsilon}_{k}\right\rangle, \\
& \frac{d}{d t} L_{\dot{x}_{k}}=\frac{1}{2}\left\langle\hat{\boldsymbol{I}} \dot{\boldsymbol{B}}^{\mathrm{T}} \omega_{\dot{\boldsymbol{x}}} \boldsymbol{\varepsilon}_{k}+\hat{\boldsymbol{I}} \boldsymbol{B}^{\mathrm{T}} \dot{\omega}_{\dot{x}} \boldsymbol{\varepsilon}_{k}, \boldsymbol{B}^{\mathrm{T}} \omega_{\dot{x}} \dot{\boldsymbol{x}}\right\rangle+\frac{1}{2}\left\langle\hat{\boldsymbol{I}} \boldsymbol{B}^{\mathrm{T}} \omega_{\dot{x}} \boldsymbol{\varepsilon}_{k}, \dot{\boldsymbol{B}}^{\mathrm{T}} \omega_{\dot{\boldsymbol{x}}} \dot{\boldsymbol{x}}+\boldsymbol{B}^{\mathrm{T}} \dot{\omega}_{\dot{x}} \dot{\boldsymbol{x}}+\boldsymbol{B}^{\mathrm{T}} \omega_{\dot{x}} \ddot{\boldsymbol{x}}\right\rangle \\
& +\frac{1}{2}\left\langle\hat{\boldsymbol{I}} \dot{\boldsymbol{B}}^{\mathrm{T}} \boldsymbol{\omega}_{\dot{x}} \dot{\boldsymbol{x}}+\hat{\boldsymbol{I}} \boldsymbol{B}^{\mathrm{T}} \dot{\omega}_{\dot{\boldsymbol{x}}} \dot{\boldsymbol{x}}+\hat{\boldsymbol{I}} \boldsymbol{B}^{\mathrm{T}} \omega_{\dot{\boldsymbol{x}}} \ddot{\boldsymbol{x}}, \boldsymbol{B}^{\mathrm{T}} \boldsymbol{\omega}_{\dot{\boldsymbol{x}}} \boldsymbol{\varepsilon}_{k}\right\rangle+\frac{1}{2}\left\langle\hat{\boldsymbol{I}} \boldsymbol{B}^{\mathrm{T}} \omega_{\dot{x}} \dot{\boldsymbol{x}}, \dot{\boldsymbol{B}}^{\mathrm{T}} \boldsymbol{\omega}_{\dot{x}} \boldsymbol{\varepsilon}_{k}+\boldsymbol{B}^{\mathrm{T}} \dot{\omega}_{\dot{x}} \boldsymbol{\varepsilon}_{k}\right\rangle \\
& =\varepsilon_{k}^{\mathrm{T}} \boldsymbol{\omega}_{\dot{\boldsymbol{x}}}^{\mathrm{T}} \dot{\boldsymbol{B}} \hat{\boldsymbol{I}} \boldsymbol{B}^{\mathrm{T}} \omega_{\dot{x}} \dot{\boldsymbol{x}}+\boldsymbol{\varepsilon}_{k}^{\mathrm{T}} \dot{\omega}_{\dot{x}}^{\mathrm{T}} \boldsymbol{B} \hat{\boldsymbol{I}} \boldsymbol{B}^{\mathrm{T}} \omega_{\dot{x}} \dot{x}+\varepsilon_{k}^{\mathrm{T}} \boldsymbol{\omega}_{\dot{\boldsymbol{x}}}^{\mathrm{T}} \boldsymbol{B} \hat{\boldsymbol{I}} \dot{\boldsymbol{B}}^{\mathrm{T}} \omega_{\dot{x}} \dot{\boldsymbol{x}} \\
& +\varepsilon_{k}^{\mathrm{T}} \omega_{\dot{x}}^{\mathrm{T}} \boldsymbol{B} \hat{\boldsymbol{I}} \boldsymbol{B}^{\mathrm{T}} \dot{\omega}_{\dot{x}} \dot{x}+\varepsilon_{k}^{\mathrm{T}} \omega_{\dot{x}}^{\mathrm{T}} \boldsymbol{B} \hat{\boldsymbol{I}} \boldsymbol{B}^{\mathrm{T}} \omega_{\dot{x}} \ddot{\boldsymbol{x}} \\
& L_{x_{k}}=\frac{1}{2}\left\langle\hat{\boldsymbol{I}} \boldsymbol{B}_{x_{k}}^{\mathrm{T}} \omega_{\dot{x}} \dot{\boldsymbol{x}}+\hat{\boldsymbol{I}} \boldsymbol{B}^{\mathrm{T}} \boldsymbol{\omega}_{\dot{\boldsymbol{x}} x_{k}} \dot{\boldsymbol{x}}, \boldsymbol{B}^{\mathrm{T}} \boldsymbol{\omega}_{\dot{\boldsymbol{x}}} \dot{\boldsymbol{x}}\right\rangle+\frac{1}{2}\left\langle\hat{\boldsymbol{I}} \boldsymbol{B}^{\mathrm{T}} \omega_{\dot{\boldsymbol{x}}} \dot{\boldsymbol{x}}, \boldsymbol{B}_{x_{k}}^{\mathrm{T}} \omega_{\dot{x}} \dot{\boldsymbol{x}}+\boldsymbol{B}^{\mathrm{T}} \omega_{\dot{\boldsymbol{x}} x_{k}} \dot{\boldsymbol{x}}\right\rangle \\
& =\dot{\boldsymbol{x}}^{\mathrm{T}}\left(\omega_{\dot{\boldsymbol{x}}}^{\mathrm{T}} \boldsymbol{B}_{x_{k}}+\omega_{\dot{\boldsymbol{x}} x_{k}}^{\mathrm{T}} \boldsymbol{B}\right) \hat{\boldsymbol{I}} \boldsymbol{B}^{\mathrm{T}} \omega_{\dot{x}} \dot{\boldsymbol{x}},
\end{aligned}
$$

where $k=1,2,3 . \quad \dot{\boldsymbol{B}}(\boldsymbol{x}, \dot{\boldsymbol{x}}) \in \mathbb{R}^{3 \times 3}$ refers to the derivative of $\boldsymbol{B}(\boldsymbol{x})$ with respect to $t$ and $\dot{\omega}_{\dot{x}}(\psi, \theta, \dot{\psi}, \dot{\theta}) \in \mathbb{R}^{3 \times 3}$ refers to the derivative of $\boldsymbol{\omega}_{\dot{\boldsymbol{x}}}(\psi, \theta) . \quad L_{\dot{x}_{k}}$ and $L_{x_{k}}$ refer to the partial derivatives of $L$ with respect to $\dot{x}_{k}$ and $x_{k}$, respectively.

Using the definition of the derivative for matrices in Ref. 19, we can easily verify that the term $\boldsymbol{\varepsilon}_{k}^{\mathrm{T}} \dot{\boldsymbol{\omega}}_{\dot{\boldsymbol{x}}}^{\mathrm{T}} \boldsymbol{B} \hat{\boldsymbol{I}} \boldsymbol{B}^{\mathrm{T}} \boldsymbol{\omega}_{\dot{\boldsymbol{x}}} \dot{\boldsymbol{x}}$ in Eq. (77) is equal to $L_{x_{k}}$ in Eq. (78) as follows:

$$
\boldsymbol{\varepsilon}_{k}^{\mathrm{T}} \dot{\boldsymbol{\omega}}_{\dot{\boldsymbol{x}}}^{\mathrm{T}} \boldsymbol{B} \hat{\boldsymbol{I}} \boldsymbol{B}^{\mathrm{T}} \boldsymbol{\omega}_{\dot{x}} \dot{\boldsymbol{x}}-\dot{\boldsymbol{x}}^{\mathrm{T}}\left(\boldsymbol{\omega}_{\dot{\boldsymbol{x}}}^{\mathrm{T}} \boldsymbol{B}_{x_{k}}+\omega_{\dot{\boldsymbol{x}} x_{k}}^{\mathrm{T}} \boldsymbol{B}\right) \hat{\boldsymbol{I}} \boldsymbol{B}^{\mathrm{T}} \boldsymbol{\omega}_{\dot{x}} \dot{\boldsymbol{x}}=0
$$

where $k=1,2,3$.

Then, we obtain the vector equations of the multirotor as follows:

$$
\omega_{\dot{x}}^{\mathrm{T}} \dot{\boldsymbol{B}} \hat{\boldsymbol{I}} \boldsymbol{B}^{\mathrm{T}} \omega_{\dot{x}} \dot{\boldsymbol{x}}+\omega_{\dot{x}}^{\mathrm{T}} \boldsymbol{B} \hat{\boldsymbol{I}} \dot{\boldsymbol{B}}^{\mathrm{T}} \omega_{\dot{x}} \dot{\boldsymbol{x}}+\omega_{\dot{x}}^{\mathrm{T}} \boldsymbol{B} \hat{\boldsymbol{I}} \boldsymbol{B}^{\mathrm{T}} \dot{\omega}_{\dot{x}} \dot{\boldsymbol{x}}+\omega_{\dot{x}}^{\mathrm{T}} \boldsymbol{B} \hat{\boldsymbol{I}} \boldsymbol{B}^{\mathrm{T}} \omega_{\dot{x}} \ddot{\boldsymbol{x}}=\omega_{\dot{x}}^{\mathrm{T}} \boldsymbol{B} \boldsymbol{F}_{\mathrm{rot}}(\boldsymbol{u})
$$

When $\left(\omega_{\dot{x}}^{T}\right)^{-1}$ is multiplied to both sides of Eq. (80) and the properties of Eq. (81) are also applied to Eq. (80), Eq. (82) is finally obtained:

$$
\begin{gathered}
\dot{\boldsymbol{B}}^{\mathrm{T}} \boldsymbol{\omega}_{\dot{x}} \dot{\boldsymbol{x}}=\dot{\boldsymbol{B}}^{\mathrm{T}} \boldsymbol{\omega}=-\boldsymbol{B}^{\mathrm{T}} \dot{\boldsymbol{B}} \boldsymbol{B}^{\mathrm{T}} \boldsymbol{\omega}=-\boldsymbol{B}^{\mathrm{T}} \boldsymbol{B}[\boldsymbol{\Omega}, \boldsymbol{\Omega}]=(0,0,0)^{\mathrm{T}}, \\
\dot{\boldsymbol{B}} \hat{\boldsymbol{I}} \boldsymbol{B}^{\mathrm{T}} \boldsymbol{\omega}_{\dot{x}} \dot{\boldsymbol{x}}+\boldsymbol{B} \hat{\boldsymbol{I}} \boldsymbol{B}^{\mathrm{T}} \dot{\boldsymbol{\omega}}_{\dot{x}} \dot{\boldsymbol{x}}+\boldsymbol{B} \hat{\boldsymbol{I}} \boldsymbol{B}^{\mathrm{T}} \omega_{\dot{x}} \ddot{\boldsymbol{x}}=\boldsymbol{B} \boldsymbol{F}_{\mathrm{rot}}(\boldsymbol{u}),
\end{gathered}
$$

where $\left(\boldsymbol{B}(\boldsymbol{x}) \hat{\boldsymbol{I}} \boldsymbol{B}(\boldsymbol{x})^{\mathrm{T}}\right): \boldsymbol{x}=(\psi, \theta, \phi) \in \mathbb{R}^{3} \rightarrow \mathbb{R}^{3 \times 3},\left(\dot{\boldsymbol{B}}(\boldsymbol{x}, \dot{\boldsymbol{x}}) \hat{\boldsymbol{I}} \boldsymbol{B}(\boldsymbol{x})^{\mathrm{T}}\right):(\boldsymbol{x}, \dot{\boldsymbol{x}})=(\psi, \theta, \phi, \dot{\psi}, \dot{\theta}, \dot{\phi}) \in \mathbb{R}^{6} \rightarrow \mathbb{R}^{3 \times 3}$.

Here, we give Theorem 1 in an explicit form with the Euler angle state variables of dynamical system equations for multirotor rotations. 
Theorem 1:

$$
\text { If } \operatorname{det}\left(\boldsymbol{B} \hat{\boldsymbol{I}} \boldsymbol{B}^{\mathrm{T}} \omega_{\dot{\boldsymbol{x}}}\right)=-I_{11} I_{22} I_{33} \cos \theta \neq 0,
$$

the dynamical system state equations for multirotor rotations are written in an explicit form in terms of Euler angle state variables $(\boldsymbol{x}, \dot{\boldsymbol{x}})^{\mathrm{T}} \in \Sigma_{\text {rot }} \in \mathbb{R}^{6}$, and the input vector function of time $\boldsymbol{u} \in \Lambda \subset \mathbb{R}^{\rho}$ controls the outer generalized forces acting on the multirotor, then

$$
\begin{gathered}
\frac{d}{d t}\left(\begin{array}{c}
\boldsymbol{x} \\
\dot{\boldsymbol{x}}
\end{array}\right)=\left(\begin{array}{c}
\dot{\boldsymbol{x}} \\
\boldsymbol{Y}(\boldsymbol{\eta}, \dot{\boldsymbol{x}})+\boldsymbol{Z}(\boldsymbol{\eta}) \boldsymbol{F}_{\mathrm{rot}}(\boldsymbol{u})
\end{array}\right), \\
\boldsymbol{Z}(\boldsymbol{\eta})=\left(\boldsymbol{B}(\boldsymbol{x}) \hat{\boldsymbol{I}} \boldsymbol{B}(\boldsymbol{x})^{\mathrm{T}} \cdot \omega_{\dot{\boldsymbol{x}}}(\psi, \theta)\right)^{-1} \cdot \boldsymbol{B}(\boldsymbol{x}), \\
\boldsymbol{Y}(\boldsymbol{\eta}, \dot{\boldsymbol{x}})=-\left(\boldsymbol{B}(\boldsymbol{x}) \hat{\boldsymbol{I}} \boldsymbol{B}(\boldsymbol{x})^{\mathrm{T}} \cdot \omega_{\dot{x}}(\psi, \theta)\right)^{-1}\left(\dot{\boldsymbol{B}}(\boldsymbol{x}, \dot{\boldsymbol{x}}) \hat{\boldsymbol{I}} \boldsymbol{B}(\boldsymbol{x})^{\mathrm{T}} \cdot \omega_{\dot{\boldsymbol{x}}}(\psi, \theta)+\boldsymbol{B}(\boldsymbol{x}) \hat{\boldsymbol{I}} \boldsymbol{B}(\boldsymbol{x})^{\mathrm{T}} \cdot \dot{\omega}_{\dot{x}}(\psi, \theta, \dot{\psi}, \dot{\theta})\right) \cdot \dot{\boldsymbol{x}},
\end{gathered}
$$

where $t \in \mathbb{R}$ refers to time, $\boldsymbol{x}=(\psi, \theta, \phi)^{\mathrm{T}}=(\psi, \boldsymbol{\eta})^{\mathrm{T}}, \boldsymbol{\eta}=(\theta, \phi)^{\mathrm{T}}, \dot{\boldsymbol{x}}=(\dot{\psi}, \dot{\theta}, \dot{\phi})^{\mathrm{T}}=(\dot{\psi}, \dot{\boldsymbol{\eta}})^{\mathrm{T}}$, $\dot{\eta}=(\dot{\theta}, \dot{\phi})^{\mathrm{T}}, \boldsymbol{\omega}=\boldsymbol{\omega}_{\dot{\boldsymbol{x}}} \dot{\boldsymbol{x}}$ and $\rho \in \mathbb{N}$.

Proof: Eq. (82) is rearranged as

$$
\left(\begin{array}{cc}
\boldsymbol{I}_{3 \times 3} & \mathbf{0}_{3 \times 3} \\
\mathbf{0}_{3 \times 3} & \boldsymbol{B} \hat{\boldsymbol{I}}^{\mathrm{T}} \boldsymbol{\omega}_{\dot{\boldsymbol{x}}}
\end{array}\right) \frac{d}{d t}\left(\begin{array}{c}
\boldsymbol{x} \\
\dot{\boldsymbol{x}}
\end{array}\right)=\left(\begin{array}{c}
\dot{\boldsymbol{x}} \\
-\left(\dot{\boldsymbol{B}} \hat{\boldsymbol{I}} \boldsymbol{B}^{\mathrm{T}} \boldsymbol{\omega}_{\dot{\boldsymbol{x}}}+\boldsymbol{B} \hat{\boldsymbol{I}} \boldsymbol{B}^{\mathrm{T}} \dot{\boldsymbol{\omega}}_{\dot{\boldsymbol{x}}}\right) \dot{\boldsymbol{x}}+\boldsymbol{B} \boldsymbol{F}_{\mathrm{rot}}(\boldsymbol{u})
\end{array}\right)
$$

where $\boldsymbol{I}_{3 \times 3}$ is the identity matrix of size 3 and $\mathbf{0}_{3 \times 3}$ is the $3 \times 3$ zero matrix.

Since the matrix on the left side of Eq. (87), $\left(\begin{array}{cc}\boldsymbol{I}_{3 \times 3} & \mathbf{0}_{3 \times 3} \\ \mathbf{0}_{3 \times 3} & \boldsymbol{B} \hat{\boldsymbol{I}} \boldsymbol{B}^{\mathrm{T}} \boldsymbol{\omega}_{\dot{\boldsymbol{x}}}\end{array}\right)$, is nonsingular, Eq. (87) can be transformed into Eq. (84). From the row-expansion formula, ${ }^{(20)}$ we obtain the following equations:

$$
\begin{gathered}
\operatorname{det}\left(\begin{array}{rr}
\boldsymbol{I}_{3 \times 3} & \mathbf{0}_{3 \times 3} \\
\mathbf{0}_{3 \times 3} & \boldsymbol{B} \hat{\boldsymbol{I}} \boldsymbol{B}^{\mathrm{T}} \omega_{\dot{\boldsymbol{x}}}
\end{array}\right)=\operatorname{det}\left(\boldsymbol{B} \hat{\boldsymbol{I}} \boldsymbol{B}^{\mathrm{T}} \omega_{\dot{\boldsymbol{x}}}\right), \\
\operatorname{det}\left(\boldsymbol{B} \hat{\boldsymbol{I}} \boldsymbol{B}^{\mathrm{T}} \boldsymbol{\omega}_{\dot{\boldsymbol{x}}}\right)=\operatorname{det}(\boldsymbol{B}) \operatorname{det}(\hat{\boldsymbol{I}}) \operatorname{det}\left(\boldsymbol{B}^{\mathrm{T}}\right) \operatorname{det}\left(\boldsymbol{\omega}_{\dot{\boldsymbol{x}}}\right)=-I_{11} I_{22} I_{33} \cos \theta .
\end{gathered}
$$

From $\operatorname{det}(\boldsymbol{B})=\operatorname{det}\left(\boldsymbol{B}^{\mathrm{T}}\right)=1, \operatorname{det}(\hat{\boldsymbol{I}})=I_{11} I_{22} I_{33}$, and $\operatorname{det}\left(\boldsymbol{\omega}_{\dot{\boldsymbol{x}}}\right)=-\cos \theta$, we find that Eq. (89) holds. Hence, if $\operatorname{det}(\hat{\boldsymbol{I}}) \neq 0$ and $\theta \neq \pi / 2$ (rad), then Eq. (84) holds. Using straightforward matrix calculations, it is easily verified that the function in Eq. (85) does not have the state variables $\psi$ and $\dot{\boldsymbol{x}}$, and the function in Eq. (86) does not have the state variable $\psi$. Thus, from the preceding discussion, Theorem 1 is proved. 


\subsubsection{Equations of translation}

Taking variables $\boldsymbol{r}$ and $\dot{\boldsymbol{r}}$ as the state variables of the multirotor translations, we derive an Euler-Lagrange equation of translation for the multirotor as follows:

$$
\frac{d}{d t} L_{\dot{r}}(\dot{\boldsymbol{r}})-L_{\boldsymbol{r}}=\boldsymbol{B}(\boldsymbol{x}) \boldsymbol{F}_{\mathrm{tra}}(\boldsymbol{u})
$$

where $\boldsymbol{B}(\boldsymbol{x}) \boldsymbol{F}_{\operatorname{tra}}(\boldsymbol{u}), \boldsymbol{F}_{\operatorname{tra}}(\boldsymbol{u})=\boldsymbol{S}_{\operatorname{tra} 2 p} \boldsymbol{u}, \boldsymbol{u} \in \Lambda$ refers to the generalized forces (so-called thrusts). Therefore, we obtain a vector equation of translation for the multirotor as follows:

$$
\ddot{\boldsymbol{r}}=-g \boldsymbol{e}_{3}+\frac{1}{m} \boldsymbol{B}(\boldsymbol{x}) \boldsymbol{F}_{\mathrm{tra}}(\boldsymbol{u}) .
$$

We summarize the dynamical state equation of translation for the multirotor as follows:

$$
\frac{d}{d t}\left(\begin{array}{c}
\boldsymbol{r} \\
\dot{\boldsymbol{r}}
\end{array}\right)=\left(\begin{array}{c}
\dot{\boldsymbol{r}} \\
-g \boldsymbol{e}_{3}+\frac{1}{m} \boldsymbol{B}(\boldsymbol{x}) \boldsymbol{F}_{\text {tra }}(\boldsymbol{u})
\end{array}\right)
$$

On the basis of the above equations, we propose Theorem 2 as an explicit form of dynamical system equations of translation for the multirotor as follows.

Theorem 2: Let $(\boldsymbol{x}(t), \dot{\boldsymbol{x}}(t))^{\mathrm{T}}$ be the solution for Eq. (84) in Theorem 1. Then, the dynamical system state equation of translation for the multirotor is obtained in the following explicit form:

$$
\frac{d}{d t}\left(\begin{array}{c}
\boldsymbol{r} \\
\dot{\boldsymbol{r}}
\end{array}\right)=\left(\begin{array}{c}
\dot{\boldsymbol{r}} \\
-g \boldsymbol{e}_{3}+\frac{1}{m} \boldsymbol{B}\left(\phi_{1}\left(t,\left(\boldsymbol{x}_{0}, \dot{\boldsymbol{x}}_{0}\right)^{\mathrm{T}}, \boldsymbol{u}\right)\right) \boldsymbol{F}_{\mathrm{tra}}(\boldsymbol{u})
\end{array}\right)
$$

where $(\boldsymbol{x}(t), \dot{\boldsymbol{x}}(t))^{\mathrm{T}}=\left(\boldsymbol{\phi}_{1}\left(t,\left(\boldsymbol{x}_{0}, \dot{\boldsymbol{x}}_{0}\right)^{\mathrm{T}}, \boldsymbol{u}\right), \boldsymbol{\phi}_{2}\left(t,\left(\boldsymbol{x}_{0}, \dot{\boldsymbol{x}}_{0}\right)^{\mathrm{T}}, \boldsymbol{u}\right)\right)^{\mathrm{T}}=\boldsymbol{\phi}\left(t,\left(\boldsymbol{x}_{0}, \dot{\boldsymbol{x}}_{0}\right)^{\mathrm{T}}, \boldsymbol{u}\right) \in \Sigma_{\text {rot }} \subset \mathbb{R}^{6}$ with initial points $\left(t_{0},\left(\boldsymbol{x}_{0}, \dot{\boldsymbol{x}}_{0}\right)^{\mathrm{T}}\right) \in \mathbb{R} \times \Sigma_{\text {rot }} \subset \mathbb{R} \times \mathbb{R}^{6}$ and $\boldsymbol{u} \in \Lambda$.

Proof: The dynamical state equation of translation for the multirotor clearly comes from Eq. (92). Since $\boldsymbol{x}$ of $\boldsymbol{B}(\boldsymbol{x})$ in Eq. (92) is definitely given by the solution $\boldsymbol{\phi}_{1}\left(t,\left(\boldsymbol{x}_{0}, \dot{\boldsymbol{x}}_{0}\right)^{\mathrm{T}}, \boldsymbol{u}\right)$ of Eq. (84), Eq. (92) is replaced with Eq. (93). Thus, Theorem 2 is proved.

\section{Appendix 2: Theorem to Achieve Multirotor Maneuvers and Flight States of Fig.} 5 in Table 2

Theorem 3: Let $\boldsymbol{F}\left(\boldsymbol{x}, \boldsymbol{u}_{2 p}\right)$ be the following $C^{1}$ function taking values in $\mathbb{R}^{2 p}, p=2,3$, 4 with a neighborhood of Euler angle state variables $\tilde{\boldsymbol{x}}$ in $\mathbb{R}^{3}$ and the motor speed control signal vector $\tilde{\boldsymbol{u}}_{2 p}$ in $\mathbb{R}^{2 p}$ with, $\boldsymbol{F}\left(\boldsymbol{x}, \boldsymbol{u}_{2 p}\right)=\mathbf{0}_{2 p}=(0,0, \ldots, 0)^{\mathrm{T}}$ including both lower sections of $\boldsymbol{Z}\left(\boldsymbol{\eta}_{\mathrm{op}}\right) \boldsymbol{S}_{\text {rot } 2 p} \boldsymbol{u}_{2 p(\mathrm{op})}$ in Eq. (16) and $\left\langle\boldsymbol{e}_{3,}-g \boldsymbol{e}_{3}+1 / m \boldsymbol{B}\left(\boldsymbol{x}_{\mathrm{op}}\right) \boldsymbol{S}_{\text {tra } 2 p} \boldsymbol{u}_{2 p(\mathrm{op})}\right\rangle$ in Eq. (17): 


$$
\boldsymbol{F}\left(\boldsymbol{x}, \boldsymbol{u}_{2 p}\right)=\boldsymbol{A}_{2 p}(\boldsymbol{x}) \boldsymbol{u}_{2 p}-\boldsymbol{b}_{2 p} .
$$

In the case of $p=2$,

$$
\boldsymbol{A}_{4}(\boldsymbol{\eta}) \in \mathbb{R}^{4 \times 4}=\left(\begin{array}{c}
\boldsymbol{Z}(\boldsymbol{\eta}) \boldsymbol{S}_{\mathrm{rot} 4} \\
\boldsymbol{e}_{3}^{\mathrm{T}} \frac{1}{m} \boldsymbol{B}(\boldsymbol{x}) \boldsymbol{S}_{\mathrm{tra} 4}
\end{array}\right)
$$

where $c \in \mathbb{R}$ is a constant and $\boldsymbol{b}_{4}=(\ddot{\psi}, \ddot{\theta}, \ddot{\phi}, c+g)^{\mathrm{T}} \in \mathbb{R}^{4}$.

In the case of $p=3$ or 4 ,

$$
\begin{gathered}
\boldsymbol{A}_{2 p}(\boldsymbol{x}) \in \mathbb{R}^{2 p \times 2 p}=\left(\begin{array}{c}
\boldsymbol{A}_{4 \times 2 p}^{\prime}(\boldsymbol{\eta}) \\
\boldsymbol{Q}_{(2 p-4) \times 2 p}
\end{array}\right), \\
\boldsymbol{A}_{4 \times 2 p}^{\prime}(\boldsymbol{\eta}) \in \mathbb{R}^{4 \times 2 p}=\left(\begin{array}{c}
\boldsymbol{Z}(\boldsymbol{\eta}) \boldsymbol{S}_{\mathrm{rot} 2 p} \\
\boldsymbol{e}_{3}^{\mathrm{T}} \frac{1}{m} \boldsymbol{B}(\boldsymbol{x}) \boldsymbol{S}_{\mathrm{tra} 2 p}
\end{array}\right),
\end{gathered}
$$

where $\boldsymbol{Q}_{(2 p-4) \times 2 p} \in \mathbb{R}^{(2 p-4) \times 2 p}$ is a constant matrix, $\boldsymbol{b}_{2 p}=\left(\ddot{\psi}, \ddot{\theta}, \ddot{\phi}, c+g, \boldsymbol{b}_{2 p-4}^{\prime}\right)^{\mathrm{T}} \in \mathbb{R}^{2 p}$, and $\boldsymbol{b}_{2 p-4}^{\prime} \in \mathbb{R}^{2 p-4}$ is a constant vector.

If for arbitrary $\tilde{\boldsymbol{x}} \in \mathbb{R}^{3}, \operatorname{det}\left(\boldsymbol{A}_{2 p}(\tilde{\boldsymbol{x}})\right) \neq 0$, and $\tilde{\boldsymbol{u}}_{2 p} \in \mathbb{R}^{2 p}$, such that $\boldsymbol{F}\left(\tilde{\boldsymbol{x}}, \tilde{\boldsymbol{u}}_{2 p}\right)=0_{2 p}$, then $\tilde{\boldsymbol{u}}_{2 p}=\boldsymbol{A}_{2 p}(\tilde{\boldsymbol{x}})^{-1} \boldsymbol{b}_{2 p}$ is uniquely obtained. Then, $\ddot{\psi}, \ddot{\theta}$, and $\ddot{\boldsymbol{\phi}}$ are also determined as

$$
\begin{aligned}
\ddot{\psi} & =\left\langle\boldsymbol{\varepsilon}_{1}, \boldsymbol{Y}(\boldsymbol{\eta}, \dot{\boldsymbol{x}})+\boldsymbol{Z}(\boldsymbol{\eta}) \boldsymbol{S}_{\mathrm{rot} 2 p} \boldsymbol{u}_{2 p}\right\rangle, \\
\ddot{\theta} & =\left\langle\boldsymbol{\varepsilon}_{2}, \boldsymbol{Y}(\boldsymbol{\eta}, \dot{\boldsymbol{x}})+\boldsymbol{Z}(\boldsymbol{\eta}) \boldsymbol{S}_{\mathrm{rot} 2 p} \boldsymbol{u}_{2 p}\right\rangle, \\
\ddot{\phi} & =\left\langle\boldsymbol{\varepsilon}_{3}, \boldsymbol{Y}(\boldsymbol{\eta}, \dot{\boldsymbol{x}})+\boldsymbol{Z}(\boldsymbol{\eta}) \boldsymbol{S}_{\mathrm{rot} 2 p} \boldsymbol{u}_{2 p}\right\rangle .
\end{aligned}
$$

Proof: In the case of $p=2$, when we set $\tilde{\boldsymbol{\eta}} \in \mathbb{R}^{2}$ as a fixed-value vector, $\boldsymbol{A}_{4}(\tilde{\boldsymbol{\eta}})$ becomes a $4 \times 4$ constant matrix.

In the case of $p=3$ or 4 , when we also set $\tilde{\boldsymbol{x}} \in \mathbb{R}^{3}\left(\tilde{\boldsymbol{x}}=(\tilde{\psi}, \tilde{\boldsymbol{\eta}})^{\mathrm{T}}\right)$ as a fixed-value vector, $A_{4 \times 2 p}^{\prime}(\tilde{\eta})$ becomes a $4 \times 2 p$ constant matrix. Furthermore, we can choose a $(2 p-4) \times 2 p$ constant matrix $\boldsymbol{Q}_{(2 p-4) \times 2 p}$ freely.

Hence, if for arbitrary $\tilde{\boldsymbol{x}} \in \mathbb{R}^{3}, \operatorname{det}\left(\boldsymbol{A}_{2 p}(\tilde{\boldsymbol{x}})\right) \neq 0$, then $\tilde{\boldsymbol{u}}_{2 p} \in \mathbb{R}^{2 p}$ is uniquely obtained as $\tilde{\boldsymbol{u}}_{2 p}=\boldsymbol{A}_{2 p}(\tilde{\boldsymbol{x}})^{-1} \boldsymbol{b}_{2 p}$. Finally, by using the fourth-, fifth-, and sixth-row equations of Eq. (12), $\ddot{\psi}, \ddot{\theta}$, and $\ddot{\phi}$ are clearly given. Thus, Theorem 3 is proved.

Note that quadrotor flight simulations using Theorem 3 were reported in Ref. 6 . Therefore, in this paper, multirotor flight simulations using Theorem 3 are omitted. 


\section{Appendix 3: Theorem to Achieve Two Types of Multirotor Flight State in Table 3 to Avoid a Crash}

In the following, $n \in \mathbb{N}$ is the number of failed motors, $1 \leq n \leq 2 p-2, p=2,4$ (or $1 \leq n \leq 2 p-3$, $p=3$ ).

Theorem 4: When some motors fail and stop in the case of the type (I) state in Table 3, let $\boldsymbol{F}\left(\boldsymbol{x}, \boldsymbol{u}_{2}^{i_{1}, i_{2}, \ldots, i_{n}}\right)$ be the following $C^{1}$ function taking values in $\mathbb{R}^{2 p-n}, p=2,3$, 4 with a neighborhood of Euler angle state variables $\tilde{\boldsymbol{x}}$ in $\mathbb{R}^{3}$ and the motor speed control signal vector of the remaining motors $\tilde{\boldsymbol{u}}_{2 p-n}^{i_{1}, i_{2}, \ldots, i_{n}}$ in $\mathbb{R}^{2 p-n}$ with $\boldsymbol{F}\left(\tilde{\boldsymbol{x}}, \tilde{\boldsymbol{u}}_{2 p-n}^{i_{1}, i_{2}, \ldots, i_{n}}\right)=0_{2 p-n}=(0,0, \ldots, 0)^{\mathrm{T}}$, including both lower sections of $\boldsymbol{Z}\left(\boldsymbol{\eta}_{\mathrm{op}}\right) \boldsymbol{S}_{\operatorname{rot} 2 p-n}^{i_{1}, i_{2}, \ldots, i_{n}} \boldsymbol{u}_{2 p-n(\mathrm{op})}^{i_{1}, i_{2}, \ldots, i_{n}}$ in Eq. (32) and $\left\langle\boldsymbol{e}_{3,},-g \boldsymbol{e}_{3}+1 / m \boldsymbol{B}\left(\boldsymbol{x}_{\mathrm{op}}\right) \boldsymbol{S}_{\text {tra2 } 2 p-n}^{i_{1}, i_{2}, \ldots, i_{n}} \boldsymbol{u}_{2 p-n(\mathrm{op})}^{i_{1}, i_{2}, \ldots, i_{n}}\right\rangle$ in Eq. (33):

$$
\boldsymbol{F}\left(\boldsymbol{x}, \boldsymbol{u}_{2 p-n}^{i_{1}, i_{2}, \ldots, i_{n}}\right)=\boldsymbol{A}_{2 p-n}^{i_{1}, i_{2}, \ldots, i_{n}}(\boldsymbol{x}) \boldsymbol{u}_{2 p-n}^{i_{1}, i_{2}, \ldots, i_{n}}-\boldsymbol{b}_{2 p-n} .
$$

In the case of $p=2$,

$$
\boldsymbol{A}_{4-n}^{i_{1}, i_{2}, \ldots, i_{n}}(\boldsymbol{\eta}) \in \mathbb{R}^{(4-n) \times(4-n)}=\left(\begin{array}{c}
\boldsymbol{Z}(\boldsymbol{\eta}) \boldsymbol{S}_{\mathrm{rot}}^{i_{1}, i_{2}, \ldots, i_{n}} \\
\boldsymbol{e}_{3}^{\mathrm{T}} \frac{1}{m} \boldsymbol{B}(\boldsymbol{x}) \boldsymbol{S}_{\text {tra } 4,-, n}^{i_{2}, \ldots, i_{n}}
\end{array}\right),
$$

where $c \in \mathbb{R}$ is a constant and $\boldsymbol{b}_{4-n}=(\ddot{\psi}, \ddot{\theta}, \ddot{\phi}, c+g)^{\mathrm{T}} \in \mathbb{R}^{4-n}$.

In the case of $p=3$ or 4 ,

$$
\begin{aligned}
& \boldsymbol{A}_{2 p-n}^{i_{1}, i_{2}, \ldots, i_{n}}(\boldsymbol{x}) \in \mathbb{R}^{(2 p-n) \times(2 p-n)}=\left(\begin{array}{c}
\boldsymbol{A}_{4 \times 2(2 p-n)}^{i_{1}, i_{2}, \ldots, i_{n}}(\boldsymbol{\eta}) \\
\boldsymbol{Q}_{(2 p-4) \times(2 p-n)}^{i_{1}, i_{2}, \ldots, i_{n}}
\end{array}\right), \\
& \boldsymbol{A}_{4 \times(2 p-n)}^{i_{1}, i_{2}, \ldots, i_{n}}(\boldsymbol{\eta}) \in \mathbb{R}^{4 \times(2 p-n)}=\left(\begin{array}{c}
\boldsymbol{Z}(\boldsymbol{\eta}) \boldsymbol{S}_{\operatorname{rot}}^{i_{1}, i_{2}, \ldots, i_{n}} \\
\boldsymbol{e}_{3}^{\mathrm{T}} \frac{1}{m} \boldsymbol{B}(\boldsymbol{x}) \boldsymbol{S}_{\text {tra } 2 p-n}^{i_{1}, i_{2}, \ldots, i_{n}}
\end{array}\right),
\end{aligned}
$$

where $\boldsymbol{Q}_{(2 p-4) \times(2 p-n)}^{i_{1}, i_{2}, \ldots, i_{n}} \in \mathbb{R}^{(2 p-4) \times(2 p-n)}$ is a constant matrix, $\boldsymbol{b}_{2 p-n}=\left(\ddot{\psi}, \ddot{\theta}, \ddot{\phi}, c+g, \boldsymbol{b}_{2 p-4}^{\prime}\right)^{\mathrm{T}} \in \mathbb{R}^{2}$, and $\boldsymbol{b}_{2 p-4}^{\prime} \in \mathbb{R}^{2 p-4}$ is a constant vector.

If for arbitrary $\tilde{\boldsymbol{x}} \in \mathbb{R}^{3}, \operatorname{det}\left(\boldsymbol{A}_{2 p-n}^{i_{1}, i_{2}, \ldots, i_{n}}(\tilde{\boldsymbol{x}})\right) \neq 0$, and $\tilde{\boldsymbol{u}}_{2 p-n}^{i_{1}, i_{2}, \ldots, i_{n}} \in \mathbb{R}^{2 p-n}$, such that $\boldsymbol{F}\left(\tilde{\boldsymbol{x}}, \tilde{\boldsymbol{u}}_{2 p-n}^{i_{1}, i_{2}, \ldots, i_{n}}\right)=\mathbf{0}_{2 p-n}$, then $\tilde{\boldsymbol{u}}_{2 p-n}^{i_{1}, i_{2}, \ldots, i_{n}}=\boldsymbol{A}_{2 p-n}^{i_{1}, i_{2}, \ldots, i_{n}}(\tilde{\boldsymbol{x}})^{-1} \boldsymbol{b}_{2 p-n}$ is uniquely obtained. Then, $\ddot{\psi}, \ddot{\theta}$, and $\ddot{\phi}$ are also determined as 


$$
\begin{aligned}
& \ddot{\psi}=\left\langle\boldsymbol{\varepsilon}_{1}, \boldsymbol{Y}(\boldsymbol{\eta}, \dot{\boldsymbol{x}})+\boldsymbol{Z}(\boldsymbol{\eta}) \boldsymbol{S}_{\mathrm{rot} 2 p-n}^{i_{1}, i_{2}, \ldots, i_{n}} \boldsymbol{u}_{2 p-n}^{i_{1}, i_{2}, \ldots, i_{n}}\right\rangle, \\
& \ddot{\theta}=\left\langle\boldsymbol{\varepsilon}_{2}, \boldsymbol{Y}(\boldsymbol{\eta}, \dot{\boldsymbol{x}})+\boldsymbol{Z}(\boldsymbol{\eta}) \boldsymbol{S}_{\mathrm{rot} 2 p-n}^{i_{1}, i_{2}, \ldots, i_{n}} \boldsymbol{u}_{2 p-n}^{i_{1}, i_{2}, \ldots, i_{n}}\right\rangle, \\
& \ddot{\boldsymbol{\phi}}=\left\langle\boldsymbol{\varepsilon}_{3}, \boldsymbol{Y}(\boldsymbol{\eta}, \dot{\boldsymbol{x}})+\boldsymbol{Z}(\boldsymbol{\eta}) \boldsymbol{S}_{\operatorname{rot} 2 p-n}^{i_{1}, i_{2}, \ldots, i_{n}} \boldsymbol{u}_{2 p-n}^{i_{1}, i_{2}, \ldots, i_{n}}\right\rangle .
\end{aligned}
$$

Thus, $\tilde{\boldsymbol{u}}_{2 p-n}^{i_{1}, i_{2}, \ldots, i_{n}}$ in $\mathbb{R}^{2 p-n}$ are obtained as the motor speed control signals to achieve flight states to avoid a crash of type (I) in Table 3.

Proof: In the case of $p=2$, when we set $\tilde{\boldsymbol{\eta}} \in \mathbb{R}^{2}$ as a fixed-value vector, $\boldsymbol{A}_{4-n}^{i_{1}, i_{2}, \ldots, i_{n}}(\tilde{\boldsymbol{\eta}})$ becomes a $(4-n) \times(4-n)$ constant matrix.

In the case of $p=3$ or 4 , when we also set $\tilde{\boldsymbol{x}} \in \mathbb{R}^{3}\left(\tilde{\boldsymbol{x}}=(\tilde{\psi}, \tilde{\boldsymbol{\eta}})^{\mathrm{T}}\right)$ as a fixed-value vector, $\boldsymbol{A}_{4 \times(2 p-n)}^{\prime i_{1}, i_{2}, \ldots, i_{n}}(\tilde{\boldsymbol{\eta}})$ becomes a $4 \times(2 p-n)$ constant matrix. Furthermore, we can choose a $(2 p-4) \times(2 p-n)$ constant matrix $\boldsymbol{Q}_{(2 p-4) \times(2 p-n)}^{i_{1}, i_{2}, \ldots, i_{n}}$ freely.

Hence, if for arbitrary $\tilde{\boldsymbol{x}} \in \mathbb{R}^{3}, \operatorname{det}\left(\boldsymbol{A}_{2 p-n}^{i_{1}, i_{2}, \ldots, i_{n}}(\tilde{\boldsymbol{x}})\right) \neq 0$, then $\tilde{\boldsymbol{u}}_{2 p-n}^{i_{1}, i_{2}, \ldots, i_{n}} \in \mathbb{R}^{2 p-n}$ is uniquely obtained as $\tilde{\boldsymbol{u}}_{2 p-n}^{i_{1}, i_{2}, \ldots, i_{n}}=\boldsymbol{A}_{2 p-n}^{i_{1}, i_{2}, \ldots, i_{n}}(\tilde{\boldsymbol{x}})^{-1} \boldsymbol{b}_{2 p-n}$. Finally, by using the fourth-, fifth-, and sixth-row equations of Eq. (30), $\ddot{\psi}, \ddot{\theta}$, and $\ddot{\phi}$ are clearly given. Thus, Theorem 4 is proved.

Theorem 5: When some motors fail and stop in the case of the type (II) state in Table 3, let $\boldsymbol{F}\left(\boldsymbol{x}, \boldsymbol{u}_{2 p-n}^{i_{1}, i_{2}, \ldots, i_{n}}\right)$ be the following $C^{1}$ function taking values in $\mathbb{R}^{2 p-n}, p=2,3$, 4 with a neighborhood of Euler angle state variables $\tilde{\boldsymbol{x}}$ in $\mathbb{R}^{3}$ and the motor speed control signal vector of the remaining motors $\tilde{\boldsymbol{u}}_{2 p-n}^{i_{1}, i_{2}, \ldots, i_{n}}$ in $\mathbb{R}^{2 p-n}$ with $\boldsymbol{F}\left(\tilde{\boldsymbol{x}}, \tilde{\boldsymbol{u}}_{2 p-n}^{\dot{i}_{1}, i_{2}, \ldots, i_{n}}\right)=\mathbf{0}_{2 p}=(0,0, \ldots, 0)^{\mathrm{T}}$, including both lower sections of $\left\langle\boldsymbol{\varepsilon}_{2}, \boldsymbol{Z}\left(\boldsymbol{\eta}_{\mathrm{op}}\right) \boldsymbol{S}_{\text {rot } 2 p-n}^{i_{1}, i_{2}, \ldots, i_{n}} \boldsymbol{u}_{2 p-n(\mathrm{op})}^{i_{1}, i_{2}, \ldots, i_{n}}\right\rangle$ and $\left\langle\boldsymbol{\varepsilon}_{3}, \boldsymbol{Z}\left(\boldsymbol{\eta}_{\mathrm{op}}\right) \boldsymbol{S}_{\operatorname{rot} 2 p-n}^{i_{1}, i_{2}, \ldots, i_{n}} \boldsymbol{u}_{2 p-n(\mathrm{o})}^{i_{1}, i_{2}, \ldots, i_{n}}\right\rangle$ in Eq. (38) and $\left\langle\boldsymbol{e}_{3},-g \boldsymbol{e}_{3}+1 / m \boldsymbol{B}\left(\boldsymbol{x}_{\mathrm{op}}\right) \boldsymbol{S}_{\text {tra2 } 2 p-n}^{i_{1}, i_{2}, \ldots, i_{n}} \boldsymbol{u}_{2 p-n(\mathrm{op})}^{i_{1}, i_{2}, \ldots, i_{n}}\right\rangle$ in Eq. (33):

$$
\boldsymbol{F}\left(\boldsymbol{x}, \boldsymbol{u}_{2 p-n}^{i_{1}, i_{2}, \ldots, i_{n}}\right)=\boldsymbol{A}_{2 p-n}^{i_{1}, i_{2}, \ldots, i_{n}}(\boldsymbol{x}) \boldsymbol{u}_{2 p-n}^{i_{1}, i_{2}, \ldots, i_{n}}-\boldsymbol{b}_{2 p-n} .
$$

In the case of $p=2$,

$$
\boldsymbol{A}_{3-n^{\prime}}^{i_{1}, i_{2}, \ldots, i_{n}}(\boldsymbol{\eta}) \in \mathbb{R}^{\left(3-n^{\prime}\right) \times(4-n)}=\left(\begin{array}{c}
\boldsymbol{\varepsilon}_{2}^{\mathrm{T}} \boldsymbol{Z}(\boldsymbol{\eta}) \boldsymbol{S}_{\operatorname{rot}}^{i_{1}, i_{2}, \ldots, i_{n}} \\
\boldsymbol{\varepsilon}_{3}^{\mathrm{T}} \boldsymbol{Z}(\boldsymbol{\eta}) \boldsymbol{S}_{\operatorname{rot}, i_{2}, \ldots, i_{n}}^{i_{1} 2 p-n} \\
\boldsymbol{e}_{3}^{\mathrm{T}} \frac{1}{m} \boldsymbol{B}(\boldsymbol{x}) \boldsymbol{S}_{\text {tra } 2 p-n}^{i_{1}, l_{2}, \ldots, i_{n}}
\end{array}\right),
$$

where $c \in \mathbb{R}$ is a constant, $\boldsymbol{b}_{3-n^{\prime}}=(\ddot{\theta}, \ddot{\phi}, c+g)^{\mathrm{T}} \in \mathbb{R}^{3-n^{\prime}}$, and $n^{\prime}=n-1$.

In the case of $p=3$ or 4 ,

$$
\boldsymbol{A}_{2 p-n}^{i_{1}, i_{2}, \ldots, i_{n}}(\boldsymbol{x}) \in \mathbb{R}^{\left((2 p-1)-n^{\prime}\right) \times(2 p-n)}=\left(\begin{array}{c}
\boldsymbol{A}_{3 \times(2, \ldots, n)}^{i_{1}, i_{2}, \ldots, i_{n}}(\boldsymbol{\eta}) \\
\boldsymbol{Q}_{(2 p-4) \times(2 p-n)}^{i_{1}, i_{2}, \ldots, i_{n}}
\end{array}\right),
$$




$$
\boldsymbol{A}_{3 \times(2 p-n)}^{i_{1}, i_{2}, \ldots, i_{n}}(\boldsymbol{\eta}) \in \mathbb{R}^{3 \times(2 p-n)}=\left(\begin{array}{c}
\boldsymbol{\varepsilon}_{2}^{\mathrm{T}} \boldsymbol{Z}(\boldsymbol{\eta}) \boldsymbol{S}_{\mathrm{rot}}^{i_{1}, i_{2}, \ldots, i_{n} p-n} \\
\boldsymbol{\varepsilon}_{3}^{\mathrm{T}} \boldsymbol{Z}(\boldsymbol{\eta}) \boldsymbol{S}_{\mathrm{rot}, i_{2}, \ldots, i_{n}}^{i_{1} p-n} \\
\boldsymbol{e}_{3}^{\mathrm{T}} \frac{1}{m} \boldsymbol{B}(\boldsymbol{x}) \boldsymbol{S}_{\mathrm{tra} 2 p-n}^{i_{1}, i_{2}, \ldots, i_{n}}
\end{array}\right),
$$

where $\boldsymbol{Q}_{(2 p-4) \times(2 p-n)}^{\dot{i}_{1}, i_{2}, \ldots, i_{n}} \in \mathbb{R}^{(2 p-4) \times(2 p-n)}$ is a constant matrix, $\boldsymbol{b}_{(2 p-1)-n^{\prime}}=\left(\ddot{\theta}, \ddot{\phi}, c+g, \boldsymbol{b}_{2 p-4}^{\prime}\right)^{\mathrm{T}}$, $\in \mathbb{R}^{(2 p-1)-n^{\prime}}$ and $\boldsymbol{b}_{2 p-4}^{\prime} \in \mathbb{R}^{2 p-4}$ is a constant vector.

If for arbitrary $\tilde{\boldsymbol{x}} \in \mathbb{R}^{3}, \operatorname{det}\left(\boldsymbol{A}_{2 p-n}^{i_{1}, i_{2}, \ldots, i_{n}}(\tilde{\boldsymbol{x}})\right) \neq 0$, and $\tilde{\boldsymbol{u}}_{2 p-n}^{\dot{1}_{1}, i_{2}, \ldots, i_{n}} \in \mathbb{R}^{2 p-n}$, such that $\boldsymbol{F}\left(\tilde{\boldsymbol{x}}, \tilde{\boldsymbol{u}}_{2 p-n}^{i_{1}, i_{2}, \ldots, i_{n}}\right)=0_{2 p-n}$, then $\tilde{\boldsymbol{u}}_{2 p-n}^{i_{1}, i_{2}, \ldots, i_{n}}=\boldsymbol{A}_{2 p-n}^{i_{1}, i_{2}, \ldots, i_{n}}(\tilde{\boldsymbol{x}})^{-1} \boldsymbol{b}_{2 p-n}$ is uniquely obtained. Then, $\ddot{\psi}, \ddot{\theta}$, and $\ddot{\phi}$ are also determined as

$$
\begin{aligned}
& \ddot{\psi}=\left\langle\boldsymbol{\varepsilon}_{1}, \boldsymbol{Y}(\boldsymbol{\eta}, \dot{\boldsymbol{x}})+\boldsymbol{Z}(\boldsymbol{\eta}) \boldsymbol{S}_{\mathrm{rot} 2 p-n}^{i_{1}, i_{2}, \ldots, i_{n}} \boldsymbol{u}_{2 p-n}^{i_{1}, i_{2}, \ldots, i_{n}}\right\rangle, \\
& \ddot{\theta}=\left\langle\boldsymbol{\varepsilon}_{2}, \boldsymbol{Y}(\boldsymbol{\eta}, \dot{\boldsymbol{x}})+\boldsymbol{Z}(\boldsymbol{\eta}) \boldsymbol{S}_{\mathrm{rot} 2 p-n}^{i_{1}, i_{2}, \ldots, i_{n}} \boldsymbol{u}_{2 p-n}^{i_{1}, i_{2}, \ldots, i_{n}}\right\rangle, \\
& \ddot{\boldsymbol{\phi}}=\left\langle\boldsymbol{\varepsilon}_{3}, \boldsymbol{Y}(\boldsymbol{\eta}, \dot{\boldsymbol{x}})+\boldsymbol{Z}(\boldsymbol{\eta}) \boldsymbol{S}_{\mathrm{rot} 2 p-n}^{i_{1}, i_{2}, \ldots, i_{n}} \boldsymbol{u}_{2 p-n}^{i_{1}, i_{2}, \ldots, i_{n}}\right\rangle .
\end{aligned}
$$

Thus, $\tilde{\boldsymbol{u}}_{2 p-n}^{i_{1}, i_{2}, \ldots, i_{n}}$ in $\mathbb{R}^{2 p-n}$ are obtained as the motor speed control signals to achieve flight states to avoid a crash of type (II) in Table 3. As the number of failed motors increases, the rank of $\boldsymbol{S}_{\operatorname{rot} 2 p-n}^{i_{1}, i_{2}, \ldots, i_{n}} \in \mathbb{R}^{3 \times(2 p-n)}$ or $\boldsymbol{S}_{\operatorname{tra} 2 p-n}^{i_{1}, i_{2}, \ldots, i_{n}} \in \mathbb{R}^{3 \times(2 p-n)}$ and the dimensionality of $\boldsymbol{u}_{2 p-n}^{i_{1}, i_{2}, \ldots, i_{n}} \in \mathbb{R}^{2 p-n}$ decrease.

Proof: In the case of $p=2$, when we set $\tilde{\boldsymbol{\eta}} \in \mathbb{R}^{2}$ as a fixed-value vector, $\boldsymbol{A}_{3, n^{\prime}}^{i_{1}, i_{2}, \ldots, i_{n}}(\tilde{\boldsymbol{\eta}})$ becomes a $\left(3-n^{\prime}\right) \times\left(3-n^{\prime}\right)$ constant matrix.

In the case of $p=3$ or 4 , when we also set $\tilde{\boldsymbol{x}} \in \mathbb{R}^{3}\left(\tilde{\boldsymbol{x}}=(\tilde{\psi}, \tilde{\boldsymbol{\eta}})^{\mathrm{T}}\right)$ as a fixed-value vector, $\boldsymbol{A}_{3 \times(2 p-n)}^{i_{1}, i_{2}, \ldots, i_{n}}(\tilde{\boldsymbol{\eta}})$ becomes a $3 \times(2 p-n)$ constant matrix. Furthermore, we can choose a $(2 p-4) \times(2 p-n)$ constant matrix $\boldsymbol{Q}_{(2 p-4) \times(2 p-n)}^{i_{1}, i_{2}, \ldots, i_{n}}$ freely.

Hence, if for arbitrary $\tilde{\boldsymbol{x}} \in \mathbb{R}^{3}, \operatorname{det}\left(\boldsymbol{A}_{2 p-n}^{i_{1}, i_{2}, \ldots, i_{n}}(\tilde{\boldsymbol{x}})\right) \neq 0$, then $\tilde{\boldsymbol{u}}_{2 p-n}^{i_{1}, i_{2}, \ldots, i_{n}} \in \mathbb{R}^{2 p-n}$ is uniquely obtained as $\tilde{\boldsymbol{u}}_{2 p-n}^{i_{1}, i_{2}, \ldots, i_{n}}=\boldsymbol{A}_{2 p-n}^{i_{1}, i_{2}, \ldots, i_{n}}(\tilde{\boldsymbol{x}})^{-1} \boldsymbol{b}_{2 p-n}$. Finally, by using the fourth-, fifth-, and sixth-row equations of Eq. (30), $\ddot{\psi}, \ddot{\theta}$, and $\ddot{\phi}$ are clearly given. Thus, Theorem 5 is proved. 